\title{
Structural basis of flagellar filament asymmetry and supercoil templating by Leptospira spirochete sheath proteins
}

\author{
Megan R. Brady ${ }^{1}$, Fabiana San Martin², Garrett E. Debs ${ }^{1}$, Kimberley H. Gibson ${ }^{3}$, Azalia Rodríguez ${ }^{4}$, Rosario \\ Durán ${ }^{4}$, Elsio A. Wunder Jr. ${ }^{5,6}$, Albert I. Ko ${ }^{5,6}$, Alejandro Buschiazzo ${ }^{2,7}$, Charles V. Sindelar ${ }^{1}$ \\ ${ }^{1}$ Department of Molecular Biophysics and Biochemistry, Yale University, New Haven, CT, USA \\ ${ }^{2}$ Laboratory of Molecular and Structural Microbiology, Institut Pasteur de Montevideo, Montevideo, Uruguay \\ ${ }^{3}$ Center for Cellular and Molecular Imaging: Electron Microscopy, Department of Cell Biology, Yale School of \\ Medicine, New Haven, CT 06520, USA \\ ${ }^{4}$ Analytical Biochemistry and Proteomics Unit, Institut Pasteur de Montevideo \& Instituto de Investigaciones \\ Biológicas Clemente Estable, Montevideo, Uruguay \\ ${ }^{5}$ Department of Epidemiology of Microbial Diseases, Yale School of Public Health, New Haven, CT, USA \\ ${ }^{6}$ Goncalo Moniz Institute, Oswaldo Cruz Foundation, Brazilian Ministry of Health, Salvador, Brazil \\ ${ }^{7}$ Integrative Microbiology of Zoonotic Agents, Department of Microbiology, Institut Pasteur, Paris, France
}

\section{Abstract}

Several Leptospira species are bacterial agents of leptospirosis, a neglected tropical disease responsible for 1 million cases and 50,000 deaths each year worldwide. Leptospira, like other members of the Spirochaeta phylum, possess specially adapted flagella that remain confined within the periplasm. These appendages drive a unique, corkscrew-like swimming style that enables efficient motility and pathogenesis. However, the composition, function, and molecular architecture of spirochetal flagellar filaments remain poorly understood. We solved single-particle cryo-EM structures of isolated Leptospira flagellar filaments, comparing the wild-type form to two mutant forms with different missing components and abrogated motilities. The structures reveal a complex proteinaceous sheath surrounding a conserved core composed of the FlaB flagellin homolog. Sheath proteins were found to fall into two distinct categories, both of which are required for motility. Filament 'coiling' proteins, FcpA and $\mathrm{FcpB}$, exert force on the filament when they bind its surface, causing the filament to stretch. In contrast, we identify sheath components FlaAP (newly discovered in this study) and FlaA2 as 'template' factors, which have little effect on filament shape by themselves, but partition the coiling proteins to one side of the filament. In this way, the two types of Leptospira sheath factors operate collectively on the flagellar filament to bend it from a 'relaxed' form associated with cell immobility, to a motilitycompetent shape that is tightly supercoiled. Our structures also indicate that core-sheath interactions are largely mediated by carbohydrate moieties from flagellin core side chain $\mathrm{O}$-glycosylations. The supercoiling mechanism presented here provides a benchmark for studies with other bacteria, for which near-atomic resolution structures of flagellar filament in native supercoiled forms, are still lacking. 


\section{Introduction}

Spirochetes constitute an ancient Gram-negative bacterial Phylum that comprises important human and animal pathogens, such as Borrelia burgdorferi (the etiologic agent of Lyme disease), Treponema pallidum (syphilis), and several species of Leptospira (which cause leptospirosis) (Paster 2010). Spirochetes have a spiral-shaped cell body and exhibit unique wavy or 'corkscrew-like' swimming motility, allowing them to drill through tissues and highly viscous environments with very high efficiency (Li, Motaleb et al. 2000). This form of locomotion is critical to their pathogenicity, as motility-deficient Leptospira are unable to infect their hosts (Lambert, Picardeau et al. 2012, Fontana, Lambert et al. 2016, Wunder, Figueira et al. 2016), making motility mutants attractive vaccine candidates (Wunder, Adhikarla et al. 2021).

Most bacteria generate translational motility through flagellar gyration, powered by rotation of the flagellar motor; torque is then transmitted through a hook to the long flagellar filament that ultimately provokes thrust (Berg and Anderson 1973, Berg 2003). In most bacteria, such as Salmonella, Campylobacter, Bacillus, and many others, these flagella are extracellular (Pead 1979, Namba and Vonderviszt 1997). In contrast, Spirochetes contain periplasmic flagella, wrapping around their cell body while completely confined between the inner and outer cytoplasmic membranes (Wolgemuth 2015).

The spirochete Leptospira has only one flagellum at both cell poles, with filaments $\sim 3-5 \mu \mathrm{m}$ long that do not overlap towards the center of the cell body (Nauman, Holt et al. 1969, Paster 2010, Picardeau 2017). Clockwise rotation of the flagellar motor distorts the end of the Leptospira cell body into a "hook" shape, while counter-clockwise rotation enforces instead a "spiral" shape. Translation will only occur if the two motors are rotating in these opposite directions, in which case the cell will move in the direction of the spiral end, exhibiting a gyrating hook towards the trailing end (Goldstein and Charon 1988, Goldstein and Charon 1990, Wolgemuth, Charon et al. 2006, Wolgemuth 2015). When purified, flagella from wild-type Leptospira spontaneously adopt a strong supercoiled conformation, resulting in a flat spiral spring architecture when observed with transmission electron microscopy (Bromley and Charon 1979, Trueba, Bolin et al. 1992, Gibson, Trajtenberg et al. 2020).

Most bacterial filaments are homopolymers that self-assemble from a single protein species, e.g. Salmonella flagellar filaments are composed of repeating subunits of the protein flagellin (FliC) (Berg 2003). In contrast, spirochete filaments are composed of several proteins: (i) FlaB, expressed as one or more isoforms in different spirochetes, constitutes the "core" of the appendage, orthologous to the allhelical D0 and D1 domains of FliC (Mitchison, Rood et al. 1991, Lin, Surujballi et al. 1997); and (ii) FlaA isoforms, thought to constitute a proteinaceous sheath that covers the filament core, and not exhibiting detectable homology to flagellar proteins from other bacteria (Brahamsha and Greenberg 1989, Ge and Charon 1997, Li, Corum et al. 2000, Wolgemuth, Charon et al. 2006).

Focusing on Leptospira, the flagellar filament contains four FlaB isoforms (FlaB1-4) and two FlaA isoforms (FlaA1, FlaA2) (Malmstrom, Beck et al. 2009, Lambert, Picardeau et al. 2012). Leptospira also express at least two additional sheath proteins not found in other spirochetes: Flagellar Coiling Proteins FcpA and FcpB, which contribute to the coiled shape of wild-type filaments (Wunder, Figueira et al. 2016, Wunder, Slamti et al. 2018). As in other bacterial flagella (Wyss 1998, Kurniyati, Kelly et al. 2017, Blum, Filippidou et al. 2019, Kreutzberger, Ewing et al. 2020, Montemayor, Ploscariu et al. 2021), it is presumed that the Leptospira filament core is glycosylated (Holzapfel, Bonhomme et al. 2020).

In addition to the pathogenic species of Leptospira (e.g., L. interrogans), there are also saprophytic species (e.g., L. biflexa). The pathogenic and saprophytic strains share $\sim 61 \%$ of genes, 
including all known flagellar genes (Picardeau, Bulach et al. 2008, Evangelista and Coburn 2010, Fouts, Matthias et al. 2016, Wunder, Figueira et al. 2016, Wunder, Slamti et al. 2018). As the saprophytic strains are easier to manipulate genetically and grow faster in culture (Picardeau, Bulach et al. 2008), $L$. biflexa has been used for some previous flagellar studies (Picardeau, Bulach et al. 2008, Sasaki, Kawamoto et al. 2018, Wunder, Slamti et al. 2018, Gibson, Trajtenberg et al. 2020).

Recently, we reported a 3D reconstruction of the native flagellar filament from Leptospira biflexa, obtained through cryo-subtomogram averaging. This structure revealed a striking asymmetric distribution of flagellar sheath proteins, with FcpA and FcpB localized to the outer curvature face of the coiled filament (Gibson, Trajtenberg et al. 2020). However, structural and functional roles of these and other sheath proteins are not well understood. This is due in part to a lack of atomic-level descriptions of the flagellar filament for Leptospira or any other spirochete.

Here we present single particle cryo-EM structures of three variants of Leptospira flagellar filaments: the wild-type filament and two mutant forms with specific sheath components deleted, purified respectively from $f c p A^{-}$and flaA2 knock-out strains. The structures provide near-atomic resolution descriptions of three previously described sheath proteins (FlaA2, FcpA, and FcpB) in situ on the filament. Moreover, we discovered evidence that several additional, previously undescribed proteins also reside in the sheath. Among these was a FlaA2-associated protein (FlaAP), whose structure was solved in situ.

Examination of the mutant filament structures revealed that sheath proteins can be divided into two distinct categories. In the $f c p A^{-}$mutant that lacks both FcpA and FcpB (Wunder, Figueira et al. 2016), 'asymmetric binders' FlaA2 and FlaAP colocalize in a single row along one side of the filament, leaving the other side bare. In contrast, 'coiling proteins' FcpA and FcpB assemble in a lattice on the filament surface and longitudinally stretch the filament.

In the wild-type filament, these sheath proteins coexist as an asymmetric assembly where the FcpA/FcpB lattice is disrupted by FlaA2 and FlaAP. We propose that the distinctive, tightly supercoiled shape of Leptospira filaments arises due to the resultant, asymmetric stretching forces exerted by $\mathrm{FcpA} / \mathrm{FcpB}$ on the filament. This mechanism explains why neither FlaA2 nor the coiling proteins are alone sufficient for motility and pathogenesis. We also describe a previously unsuspected role for glycosylated side chains from the core FlaB proteins, which were found to mediate interactions with the sheath.

\section{Results}

\section{Sheath proteins FlaA2 and FlaAP form a row localized to the filament inner curvature}

The knock-out deletion of the $f c p A$ gene results in a viable, yet non-motile Leptospira strain that forms flagellar filaments with only one known sheath factor: FlaA (Wunder, Figueira et al. 2016). The lack of FcpA precludes normal FcpB recruitment and hence the $f c p A^{-}$mutant filaments do not form the tight coils seen in purified wild-type flagella (Wunder, Figueira et al. 2016, Sasaki, Kawamoto et al. 2018).This strain may shed light onto the potential role of additional sheath proteins, such as FlaA, in determining filament shape and function. Single-particle analysis cryo-EM was performed on thin flagellar filaments from the $L$. biflexa fcpA- strain yielding a 3D reconstruction at 3.8 $\AA$ resolution (Fig. 1 and Table S1). While the filaments were heterogeneous in structure and composition, single particle refinement and classification (Fig. S1A) yielded the near-atomic resolution structure of a single dominant population 
(Fig. 1B; $~ 53 \%$ of the total number of filament segments). This structure exhibits a pronounced curvature (Fig. 1C). Moreover, two rows of sheath proteins decorate one side of the filament (the inner curvature; Fig. 1C,E), while the rest of the filament is bare. This sheath structure contrasts with wild-type filaments, which are thicker, much more tightly coiled and are enclosed by a much more extensive sheath layer (Gibson, Trajtenberg et al. 2020).

Features in the $f c p A^{-}$density maps were sufficiently well resolved (3.4-4.4 $\AA$; Fig. S1C) to build atomic models for the core region composed of the FlaB flagellin homolog, as well as for two distinct sheath proteins: $\mathrm{FlaA} 2$, a conserved spirochete sheath factor that was known to be present in this sample (Fig. 1B,D; Fig. 2A,B), and a second, unexpected protein fold that did not match any of the known sheath components of Leptospira (Fig. 1B,D; Fig. 2C,D).

While neither of these sheath proteins have previously been structurally characterized experimentally, FlaA2 could be identified based on excellent correspondence between its density features and a structure generated by the AlphaFold2 prediction software (Fig. S1D) (Jumper, Evans et al. 2021). Lower resolution limited the ability to trace and sequence the second sheath component directly from the cryo-EM maps. In order to pinpoint the identity of this second component, a mass spectrometry-based proteomics approach was followed, analyzing purified $f c p A$ - flagellar filaments (Table S2; see Methods). Approximately 100 uncharacterized proteins were thus identified in triplicate replicas from independent purifications, apart from the known filament proteins. The 3D structures of the five most abundant uncharacterized proteins were predicted with AlphaFold2, confirming that the most abundant species explained the electron density._We have named this second sheath component FlaAP (for FlaA-associated Protein), and found it to correspond to the hypothetical protein sequence encoded by gene LEPBI_I0551 (Picardeau, Bulach et al. 2008), which is present across all Leptospira species.

FlaA2 forms a jellyroll fold, with the concave side of the beta sandwich forming a pocket that faces the flagellar core surface and forms a key part of the core interface. Density for FlaA2 was sufficiently well resolved to confirm sequence-specific side chain features, notably including several positively charged side chains lining the core interface (Fig. S2A; see section on glycan binding below). FlaAP folds into a loosely packed alpha helical bundle, with several long, protruding partially ordered loops. While side chain features were not well resolved in this region, density was observed for all predicted secondary structure features from the AlphaFold2 model, except for one disordered loop.

Adjacent FlaA2 molecules form a single row following a single FlaB protofilament (protofilament \#5; Fig. 1D), directly interacting with FlaB protofilaments \#4 and \#5. FlaAP packs laterally against FlaA2 to form its own row, following the subsequent core protofilament (\#4), next to the FlaA2 row. Together, these two proteins account for all visible sheath density in the $f c p A^{-}$structure; unique features of their interactions with each other and with the FlaB core are suggestive of functional roles in recognizing flagellar asymmetry and supercoiling as further described below.

\section{Supercoiled structure of the FlaB core reveals a 'seam'}

The FlaA2 and FlaAP sheath components lie on the surface of a $120 \AA$ diameter hollow tube (the 'core'; Fig. 1B) that broadly conforms to the flagellar filament architecture previously described in Leptospira and other bacteria (Yonekura, Maki-Yonekura et al. 2003, Maki-Yonekura, Yonekura et al. 2010, Gibson, Trajtenberg et al. 2020). In Leptospira and other spirochetes, the core is composed of one or more isoforms of FlaB, a flagellin homolog (Norris, Charon et al. 1988, Li, Motaleb et al. 2000). Our 
map resolves the core assembly to $3.4 \AA$ A resolution or better (Fig. S1C), visualizing all residues of FlaB and allowing a complete atomic model to be built. As observed in other flagellar structures (Yonekura, Maki-Yonekura et al. 2003, Maki-Yonekura, Yonekura et al. 2010, Wang, Burrage et al. 2017, Blum, Filippidou et al. 2019, Kreutzberger, Ewing et al. 2020, Montemayor, Ploscariu et al. 2021), FlaB in our structure has two distinct subdomains (D0 and D1; Fig. 1D), each composed mainly of an extended bundle of alpha helices. Also as in other structures, D0 and D1 are connected by a linker composed of short loop regions. Individual FlaB molecules assemble into linear arrays (protofilaments), 11 of which pack laterally to form the full core assembly (Fig. 1D).

In contrast to previously reported flagellar filament structures (Yonekura, Maki-Yonekura et al. 2003, Maki-Yonekura, Yonekura et al. 2010, Wang, Burrage et al. 2017, Kreutzberger, Ewing et al. 2020, Montemayor, Ploscariu et al. 2021), which correspond to idealized straight helical assemblies, our $f c p A$ structure is curved and exhibits a pronounced supercoil. The shape parameters measured for our $f c p A^{-}$ structure (left-handed supercoil with pitch and diameter of $2.0 \mu \mathrm{m}$ and $0.4 \mu \mathrm{m}$ respectively) are distinctly different than those measured for the wild-type filament (pitch and diameter of $0.22 \mu \mathrm{m}$ and $0.45 \mu \mathrm{m}$ respectively) (Gibson, Trajtenberg et al. 2020). This reflects a loss in the mutant of the characteristic tight and flattened supercoils of wild-type filaments, distortions that are linked to abrogated cell motility and pathogenicity (Wunder, Figueira et al. 2016). Instead, the supercoiled shape of $f c p A^{-}$filaments approaches the 'normal' flagellar form observed in exo-flagellated bacteria such as Salmonella enterica and Escherichia coli (Fig. S3A) (Leifson 1960, Fujii, Shibata et al. 2008, Wang, Jiang et al. 2012). Detailed structural descriptions of native supercoiled flagellar filaments have previously been unattainable for these or any other bacteria. In addition to informing Leptospira motility, the $f c p A^{-}$ structure presented here thus provides an important reference for assessing general models of natural bending and supercoiling in bacterial flagella.

Due to supercoiling, strict 11 -fold helical symmetry in the core of our $f c p A^{-}$structure is broken, and the inner curvature of the supercoil undergoes specific structural changes, broadly consistent with a 'polymorphic switching' model consistent with observations reported on Salmonella flagella (MakiYonekura, Yonekura et al. 2010, Calladine, Luisi et al. 2013). A longitudinal sliding of $\sim 2 \AA$ was observed between D1 domains of protofilaments \#4 and \#5 on the inner curvature side of the filament (Fig. S3B). This sliding is associated with a conformational change in protofilament \#4 characterized by minor deformations in the D0-D1 linker that bring the D1 proximal end 2 A closer to the distal end of D0 (Fig. $\mathrm{S3B})$. The same movement also brings the D1 proximal end closer into contact with D1 of the longitudinally adjacent neighbor ( $i-11)$ in the same protofilament (Fig. S4).

Each of the above behaviors recapitulates predicted conformational changes for a transition between two main flagellin conformations (' $R$ ' type and ' $L$ ' type) that underly the polymorphic switching model. We therefore identify the conformation of FlaB in protofilament \#4 as ' $R$ ' type, while the remaining 10 protofilaments are considered ' $L$ ' type. We note, however, that flagellar supercoiling behavior in this organism is not yet demonstrated to conform to the polymorphic switching model. Indeed, the polymorphic switch model itself remains incompletely proven (see Discussion).

In our $f c p A^{-}$supercoiled structure, consistent with the polymorphic switching model, the largest structural rearrangements are concentrated along a single protofilament (Fig. S5). This results in a 'seam' that breaks the underlying 11-fold helical subunit symmetry of a straight filament during the transition to a supercoil. 


\section{FlaA2 and FlaAP localize to the seam}

The FlaA2 sheath protein straddles protofilaments \#4 and \#5, where the seam is located, making small but significant protein-protein contacts with the D1 domains of each underlying FlaB (Fig. 2B). Because protofilaments \#4 and \#5 are axially offset from each other compared with the rest of the filament (due to the 'sliding'), they present a unique binding interface for FlaA2. While the magnitude of the sliding displacement at the seam is relatively small, 1.7 $\AA$ (Fig. S5), the FlaA2-FlaB interaction surfaces are minimal and point-like (as only four FlaA2 side chains contribute; Fig. 2B). FlaA2 interactions at non-seam locations are therefore likely to be disrupted, providing an explanation for why FlaA2 localizes to the seam in our structure.

FlaAP straddles protofilaments \#3 and \#4 and has a similar footprint on these protofilaments as FlaA2 has on protofilaments \#4 and \#5 (Fig. 2D). However, unlike FLaA2, FlaAP does not straddle the seam, and its core interface does not overlap with that of FlaA2. Thus, the interface geometry of FlaAP with FlaB is altered compared to that of FlaA2.

\section{FlaA2 and FlaAP form a lattice interconnected by flexible loops}

Neighboring FlaA2 subunits along a protofilament are connected by cable-like features. These cables, or tentacles, correspond to the FlaA2 C-terminus (residues 226-241), which projects from the edge of the jellyroll beta-sandwich pointing towards the distal end of the filament and extends unsupported over $\sim 15 \AA$ to bridge the gap between adjacent FlaA2 molecules (Fig. 3C). Residues 231-241 occupy density on the neighboring FlaA2 subunit, with the terminal residue $\left(\operatorname{Trp}_{241}\right)$ located in a hydrophobic pocket within that adjacent monomer. An additional longitudinal contact is formed by a partially ordered loop (residues 178-196) which presents the side chain of $\operatorname{Arg}_{188}$ to that of $\operatorname{Tyr}_{144}$ in the next distal FlaA2 subunit to form a probable cation-pi interaction (Fig. 3A).

Longitudinal contacts are also observed between adjacent FlaAP monomers, whose alpha helical bundles directly abut each other (Fig. 3B). Contacts involve only a few side chains, but these tend to be bulky and hydrophobic. An additional longitudinal contact is mediated by a pair of long and flexible loops that pair $\mathrm{Tyr}_{343}$ with $\mathrm{Tyr}_{174}$ of the next proximal neighbor.

FlaA2 and FlaAP also directly contact each other, via three long and meandering loops of FlaAP. Lower resolution of these loop regions in our map is consistent with greater mobility, but at least two specific contacts are identified in the map, involving clusters of mostly hydrophobic contacts (Fig. 3D). These contacts complete a lattice of nearest neighbor interactions that hold the FlaA2-FlaAP sheath assembly together. As we have described, this lattice is characterized by sparse hydrophobic interactions involving extended and flexible loops. This structural characteristic suggests that the FlaA2FlaAP assembly may be able to accommodate a variety of filament curvatures.

\section{FlaA2 and FlaAP interact with glycosylated surface residues of FlaB4}

Glycosylated residues on the FlaB core surface were observed to contribute extensively to the core-sheath interfaces for both FlaA2 and FlaAP (Fig. 2A,C). Our map directly visualizes excess densities corresponding to sites of glycosylation (Kurniyati, Kelly et al. 2017, Holzapfel, Bonhomme et al. 2020). These large, elongated densities originate from serine and threonine FlaB side chains, and many of them directly contact the sheath proteins. The latter observation contrasts with previously reported flagellar structures (Echazarreta, Kepple et al. 2018, Kreutzberger, Ewing et al. 2020), where a structural role for glycans has not been observed. While the FlaB glycans in Leptospira have not yet been chemically 
characterized, we infer that they are composed of an extended polysaccharide chain based on (1) their sizes and shapes in our map; and (2) the propensity of these glycans to simultaneously interact with multiple positively charged side chains from their protein partners.

Glycan interactions at the sheath-core interface are dominated by bridging interactions involving positively charged side chains (Lys, His, Arg) from FlaA2 and FlaAP (Fig. 2A,C). Our map identified four such contacts for FlaA2, including a prominent one involving glycosylated Ser $_{126}$. This glycan projects into a shallow pocket formed by the concave beta-sheet face of FlaA2, where it contacts $\operatorname{Arg}_{117}$ and Lys ${ }_{151}$ (Fig. 2A). The three remaining glycans surround the periphery of FlaA2 and clasp its exterior, each of them engaging a pair of FlaA2 side chains (Fig. 2A). One of these glycans, at site Ser ${ }_{187}$, also contacts residues on the proximally adjacent FlaA2 subunit, thus bridging the two subunits (Fig. 3A).

Positively charged residues of FlaAP (Lys, His) interact with the glycans of FlaB4 in a manner like that seen with FlaA2. However, while the FlaA2 interactions generally involve two FlaA2 side chains per glycan, for FlaAP we identified only one side chain per glycan interaction (Fig. 2C). Another difference with FlaA2 is that FlaAP uses glycan interactions to straddle two protofilaments in addition to proteinprotein interactions (Fig. 2C,D).

Patterns of core glycosylation in our map provide a unique fingerprint, indicating that, of the four FlaB isoforms present in Leptospira, FlaB4 predominates. Seven surface-exposed serine and threonine residues of each subunit exhibit signs of glycosylation. Two of these glycosylation sites (Thr ${ }_{137}$ and $\mathrm{Thr}_{182}$ ) are substituted in the other FlaB isoforms by residues which are not amenable to glycosylation (Gln, Glu, Asp at 137 and an Asn, lle, Glu at 182). Features consistent with the FlaB4 glycosylation fingerprint are conserved across all 11 protofilaments in our asymmetric $f c p A^{-}$ reconstruction. These features suggest that the core is predominantly composed of FlaB4 in this $L$. biflexa mutant. Mass spectrometry of the purified $f c p A^{-}$flagella detected a mixed population of FlaB isoforms (Table S2), which would be averaged together in our 3D map. FlaB4 and FlaB1 were identified as the largest isoform populations in this mutant, with a statistically insignificant difference between them (Table S2).

Both sheath protein structures show signs of being able to preferentially bind FlaB4 over the other FlaB isoforms. For FlaA2, of the four glycan contacts, three involve Ser/Thr sites conserved among all four FlaB isoforms, but the remaining site $\left(\mathrm{Thr}_{137}\right)$ is not conserved and is amenable to glycosylation only in FlaB4. This feature suggests that FlaA2 may preferentially recognize FlaB4, an idea that is further supported by the isoform-specific nature of the three FlaA2-FlaB4 protein-protein contacts observed in our structure. For the contact between $\mathrm{Val}_{180}$ (FlaB4) and $\mathrm{Phe}_{182}$ (FlaA2) (Fig. 2B), $\mathrm{Val}_{180}$ is not conserved and is located at the tip of a loop (175-184) that is shortened by 1-2 residues in the other FlaB isoforms. The second protein-protein contact, between $\mathrm{His}_{208}$ (FlaA2) and $\mathrm{Thr}_{146}$ (FlaB4), also suggests a preference for FlaB4, as in the other isoforms $\mathrm{Thr}_{146}$ is either absent or replaced by a lysine or alanine (Fig. 2B). Finally, for the contact between $\mathrm{PrO}_{66}$ of FlaA2 and $\mathrm{Met}_{157}$ of FlaB4, $\mathrm{PrO}_{66}$ is substituted in FlaB3 and FlaB2 with a glycine and an alanine respectively, likely attenuating the interaction strength (Fig. 2B). Similarly for FlaAP, of the four glycan interactions, two of these involve glycans only present in FlaB4 (Ser ${ }_{182}$, on two adjacent protofilaments). Thus, the majority of glycan contacts by FlaA2 or FlaAP show evidence of specificity for FlaB4.

\section{Purified flaA2 flagella have a straight, not curved, morphology}

A previously generated mutation of Leptospira interrogans, flaA2', was found to be non-motile and with altered flagellar filament morphology (Lambert, Picardeau et al. 2012). Similar to $f c p A^{-}$and 
$f c p B^{-}$knockouts, flagella from the flaA2 mutant were much straighter than in the wild type. However, we discovered that the coiling proteins FсpA and FсpB were both present in flaA2- filaments, indicating that the 'coiling' function of FcpA and FcpB depends on FlaA2 being present.

To examine the basis of this functional behavior, we performed cryo-EM structure analysis on purified $L$. interrogans flaA2 flagellar filaments. Cryo-EM micrographs revealed that these filaments were highly heterogeneous both in their diameters and curvatures (Fig. S10). A minority of the filaments ( 15\%) appeared nearly straight, with a diameter $\sim 24 \mathrm{~nm}$, while the majority ( $\sim 3 \%)$ of the imaged filaments were curved and thinner in nature $(\sim 12 \mathrm{~nm})$, likely reflecting a sheath-less FlaB core. Around $2 \%$ of the filaments were intermediate in both diameter $(\sim 20 \mathrm{~nm})$ and curvature, appearing to contain a partial sheath. We analyzed the straight $\sim 24 \mathrm{~nm}$ thick filaments, which provided a straightforward route to 3D structure analysis due their helically symmetric structure.

\section{FcpA and FcpB can form a helical lattice that completely encloses the flaA2 filament core}

A near-atomic resolution 3D structure of the $L$ interrogans flaA2- filament was obtained using cryoSPARC (Punjani, Rubinstein et al. 2017), applying the 11-protofilament helical symmetry common to bacterial flagella (see Methods) (Beatson, Minamino et al. 2006, Kreutzberger, Ewing et al. 2020). The resulting structure achieved an overall resolution of $2.9 \AA$, revealing a core as well as two distinct sheath layers, both of which completely enclose the FlaB core and follow its symmetry (Fig. 4). This high resolution allowed us to unambiguously identify the inner-most sheath layer as FcpA and the outer-most sheath layer as FcpB. The relationship of these sheath proteins with the core is similar to that observed in the wild-type filament (Gibson, Trajtenberg et al. 2020).

Each FcpA monomer is composed of 10 helices arranged in a ' $Y$ ', closely matching the crystal structure (San Martin, Mechaly et al. 2017, Gibson, Trajtenberg et al. 2020): one arm is formed by two long helices $(\alpha 3,4)$, and the other arm is formed by a helical bundle ( $\alpha 6-10)$. Each FcpA contacts two adjacent FlaB protofilaments as well as six FcpA molecules (two on the same protofilament, and two on each neighboring protofilament) (Fig. 5A).

Numerous longitudinal interactions occur within each FcpA row. Residues 65-96 of one FcpA monomer (located on $\alpha 1-2$ ) interacts extensively with the proximal FcpA, including an especially prominent hydrophobic interaction between $\operatorname{Trp}_{78}$ (on the $\alpha 1-2$ loop) and the $\alpha 7-8$ segment (residues 225-240) of the next proximal FcpA monomer (Fig. 5E). This N-terminal region of FcpA within the assembly is starkly different from the crystal structure, with $\alpha 1$ repositioned $\sim 52 \AA$ in the proximal direction (Fig. S6C). This displacement removes $\alpha 1$ from a hydrophobic binding pocket on the same FcpA protomer, as seen in the crystal structure, and places it instead within the corresponding pocket of the next longitudinally adjacent FcpA neighbor. Similar to a domain swap, this $\alpha 1$ rearrangement tightly links FcpA subunits together within each individual row. Additional contacts are formed between an FcpA monomer and two FcpAs located on each longitudinally adjacent row. This network of FcpA interactions in the flaA2 filament forms a strong, symmetric FcpA lattice around the entire FlaB core.

The outer-most sheath layer is completely accounted for by $\mathrm{FcpB}$, which has two helices and a seven-stranded beta-sheet, similar to the crystal structure (Fig. S6D). FcpB forms rows which overlie two FcpA rows. FcpB protomers associate longitudinally within each row but are widely separated from adjacent $\mathrm{FcpB}$ rows . Longitudinal contacts are characterized by extensive interactions between a long, C-terminal helix of one $\mathrm{FcpB}\left(\mathrm{FcpB}_{1}\right)$ with the beta sheets and helix of the distal neighboring monomer $\left(\mathrm{FcpB}_{2}\right)$ (Fig. 5C,F). These interactions are supported by additional contacts of extended loops from both monomers. The involvement of a long, relatively incompressible alpha helix in these extensive longitudinal FсpB interactions may impart rigidity to this region of the sheath. This implicit mechanical stability contrasts with the relatively flexible longitudinal interactions ('tentacles') observed for FlaA2 in 
our $f c p A^{-}$structure. These differences are likely important for the contrasting functions we infer for FlaA2 vs FcpB (see below).

There are extensive contacts between $\mathrm{FcpA}$ and $\mathrm{FcpB}$, with each FcpB interacting with five underlying FcpA monomers (Fig. 5A). Minor contacts are made between $\mathrm{FcpB}_{2}$ and three of the underlying $\mathrm{FcpAs}\left(\mathrm{FcpA}_{1}, \mathrm{FcpA}_{3}\right.$, and $\left.\mathrm{FcpA} \mathrm{A}_{5}\right)$, while major interactions occur with $\mathrm{FcpA} \mathrm{A}_{2}$ and $\mathrm{FcpA}_{4}$ (Fig. 5D). This extensive lattice network of FcpA and FcpB aids in the formation of a stable sheath layer, with correspondingly high local resolution in this sheath region (3.0 - 3.3 $\AA$ ) (Fig. S6B).

\section{Core interactions of FcpA and FcpB coiling proteins are mediated by FlaB1 glycosylation sites}

The core region of the flaA2 filament density closely resembles the core structure found in our $f c p A^{-}$mutant, except that it is straight and helically symmetric rather than curved (Fig. 4B). Thus, no 'seam' is present. Five large, globular densities, corresponding to sites of glycosylation, were seen in each FlaB monomer. The five glycosylated residues observed in the flaA2 mutant are also the location of glycan modifications in $f c p A^{-}$flagella, although the $f c p A^{-}$filaments contained two additional sites of glycosylation (the FlaB4-specific $\mathrm{Thr}_{137}$ and $\mathrm{Ser}_{182}$ ).

The glycan fingerprint of the flaA2 filaments did not allow for unambiguous FlaB isoform identification (as was possible in the $f c p A^{-}$filaments), as three of the FlaB isoforms have a Ser/Thr underlying each of the five sites of glycosylation. Instead, density at a divergent loop (residues 176-183) and at unique bulky residues in each isoform identified FlaB1 as the predominant isoform in these mutant filaments; as in the $f c p A^{-}$core, there is likely a mixture of isoforms, with the dominant isoform providing the strongest signal.

Three sheath-glycan contacts are made between each FcpA and FlaB; two of the FcpA residues are tryptophans, likely reflecting pi-stacking with the glycan sugars (Fig. 4C) (Samanta and Chakrabarti 2001). These FcpA-glycan interactions occur on both of the underlying FlaB protofilaments. One extended loop of FcpB (residues 111-124) interacts with the FlaB core, with one positively-charged FcpB residue contacting a core glycan.

Few protein-protein contacts are present between the Fcp sheath proteins and the underlying FlaB core. One contact is made between FcpB and FlaB residues, involving the same extended loop that contacts the core glycan. Five protein-protein contacts are made between FcpA and FlaB, across two FlaB protofilaments (Fig. 4D); similar to the FlaA2/FlaB4 interactions, these contacts are mostly hydrophobic in nature.

\section{Coiling proteins elongate the helical lattice of the filament}

We measured the longitudinal repeat spacing of wild-type and mutant structures by taking the center of mass of the FlaB protomers. The average spacing for each filament remains close to constant

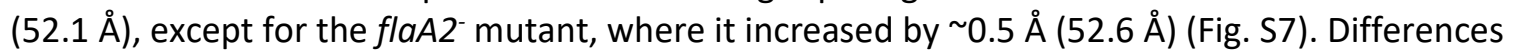
between the longitudinal spacings at the inner curvature and the outer curvature directly correlate with the overall filament curvature, with the largest spacing difference in the tightly coiled wild-type sample ( 51.1 vs $52.9 \AA$ ); the $f c p A^{-}$filaments are less curved, and thus have a smaller spacing difference ( 51.9 vs $52.2 \AA$ ). While the flaA2 mutant structure is straight, the overall filament spacing is increased to a value that exceeds any part of the $f c p A^{-}$mutant structure and approaches that of the wild-type filament outer curvature. Taken together, these observations indicate that decoration by the coiling proteins in the absence of FlaAs/FlaAP, stretch and straighten the core filament.

\section{FlaA2/FlaAP and FcpA/FcpB segregate to opposite sides of the wild-type filament}


The structure of the Leptospira wild-type flagellum has been challenging to solve at high resolution due to the flattened supercoiling geometry which traps filaments in a preferred orientation in the specimen ice layer(Gibson, Trajtenberg et al. 2020). Previously, we resorted to cryo-subtomogram averaging to overcome this obstacle, but the resolution of the resulting structure was highly anisotropic and limited to 1 nanometer at best (Gibson, Trajtenberg et al. 2020). However, we serendipitously discovered spontaneous filament fragmentation in aged wild-type specimens. The resultant shorter pieces more freely reoriented in the cryo-EM grids (see Methods). By collecting image data sets of $45^{\circ}$ tilted samples, we were therefore able to collect sufficient views to solve an isotropic reconstruction of the wild-type filament using single-particle cryo-EM methods (Fig. 6; see Methods).

The resolution achieved in the $3 \mathrm{D}$ reconstruction ( $6.5 \AA$ in the best resolved regions) allowed unambiguous fitting of $\mathrm{FlaB}, \mathrm{FcpA}, \mathrm{FcpB}$, FlaA2, and FlaAP. The overall architecture of the core and sheath proteins is consistent with the previously reported structure obtained by cryo subtomogram averaging (Gibson, Trajtenberg et al. 2020). Moreover, the detailed structure and interfaces of the coiling proteins F $\mathrm{cpA}$ and $\mathrm{FcpB}$ are consistent with our new high-resolution flaA2 mutant structure; similarly, the detailed structure interfaces of FlaA2 and FlaAP are consistent with our high-resolution $f c p A^{-}$structure.

In contrast to the flaA2 structure, however, in the wild-type structure the FcpA/FcpB helical lattice is incomplete and asymmetric. These coiling proteins are completely displaced in 5 protofilaments by FlaA2, FlaAP, as well as several additional globular densities, which likely correspond to extra sheath proteins like FlaA1 and others not yet identified. The position of FlaA2 and FlaAP on the inner curvature of the wild-type filament approximately matches that found in our $f c p A^{-}$structure. In sum, comparison of our new wild-type and mutant structures indicates that FlaA2, FlaAP, and additional unidentified components displace part of the $\mathrm{FcpA} / \mathrm{FcpB}$ helical lattice in the wild-type structure (gray regions in Fig. 6).

\section{Discussion}

Here we have presented three Leptospira filament structures: one from wild-type flagella, and two from specific sheath protein KO mutants $\left(f c p A^{-}\right.$and flaA2 $\left.{ }^{-}\right)$. The structures show evidence of FlaB glycosylation, which appears to be crucial for sheath protein binding. When the Fcp coiling factors are missing (as in the $f c p A^{-}$mutant), the sheath proteins bind only to the inner curvature of the core; in contrast, when the FlaA2 sheath factor is missing (as in the flaA2 mutant), the coiling factors bind symmetrically around the entire core. This supports a role of FlaA2 as a 'templating' factor, preventing the Fcp sheath proteins from binding along the inner curvature and segregating the 'templating' and 'coiling' factors to opposing sides of the wild-type filament (Fig. 7).

\section{Polymorphic switching in the supercoiled filament}

The curved nature of the $f c p A^{-}$filaments suggests that each FlaB protofilament will have variations from its neighbor. However, we found that one protofilament (\#4) exhibited a stark difference, with a $\sim 2 \AA$ lateral shift in the D1 domain relative to the other ten protofilaments (Fig. 7B). These other protofilaments resemble ' $L$ '-type conformations observed previously in other bacteria, while the shifted protofilament resembles the ' $R$ '-type (Wang, Burrage et al. 2017). These observations suggest that the $f c p A^{-}$mutant supercoiled form may contain a 10L/1R composition. The FlaA2 and FlaAP sheath factors are found along this ' $R$ ' protofilament, suggesting that this ' $R$ ' transition may be crucial 
for FlaA recruitment and binding (Fig. 7C). Further work will be required to determine the polymorphic form of the wild-type core, as it is probable that it too may contain a mixture of ' $L$ ' and ' $R$ ' states.

\section{Distinct functional roles of 'templating' versus 'coiling' sheath proteins}

The FlaA sheath factors appear to 'template' the core, preventing the binding of the remaining sheath proteins to the occupied inner curvature. The presence of these localized proteins ensures that the filaments will adopt a curved form, as either a 'relaxed' supercoil (when the Fcp proteins are absent; Fig. 7C) or a tight supercoil (when the Fcp proteins are also present; Fig. 7D). This role suggests that these FlaA proteins are recruited to the growing filament before the Fcp proteins, allowing them to bind along the inner curvature of the otherwise unoccupied FlaB core.

However, when the FlaA proteins are absent, FcpA and FcpB coiling factors are able to bind symmetrically around the filament, forcing the filament into a straight form (Fig. 7E). By 'stretching' the lattice, these coiling proteins thereby increase the lattice spacing of the core in both the flaA2 filaments and along the outer curvature of the wild-type filaments. Stretching along the outside of the wild-type filament would induce the formation of tight coils associated with motility. FcpA and FcpB coiling factors have not been identified in other spirochetes and may be unique to Leptospira (Wunder, Figueira et al. 2016, Wunder, Slamti et al. 2018). This observation correlates with the fact that purified flagella from other spirochetes are curved, but do not form tight supercoils. It thus remains unclear what function(s) FlaA may perform in other spirochetes, despite its widespread conservation across this phylum.

\section{Role of glycosylation in sheath factor recruitment}

We were able to structurally identify seven key glycosylation sites within the FlaB core of the $f c p A^{-}$filament, four of which appeared crucial for interactions with the FlaA2 sheath, three which appeared crucial for interactions with FlaAP, and two that did not appear to influence sheath binding. Of the five glycans in the flaA2 structure, three are involved in sheath binding (two for FcpA, and one for FcpB). It is probable that the core of the wild-type filament is similarly glycosylated, though these features cannot be resolved with the current structure. Three Leptospira glycosylation sites $\left(\operatorname{Ser}_{115}\right.$, Ser $_{126}, \mathrm{Thr}_{137}$ ) were also identified as locations of glycosylation in the FlaB core of $T$. denticola (Kurniyati, Kelly et al. 2017); while Ser $_{115}$ and Ser $_{126}$ are present in all Leptospira FlaB isoforms, $\operatorname{Thr}_{137}$ is only amenable to glycosylation in FlaB4, and is not present in our flaA2 structure. In both Leptospira and T. denticola, two of these glycosylation sites lie within the consensus sequence predicted to bind the flagellin-recognizing toll-like receptor 5 (TLR5), and glycosylation therefore may interfere with its ability to recognize the flagellum (Kurniyati, Kelly et al. 2017, Holzapfel, Bonhomme et al. 2020), though the overall periplasmic location and the sheathed character of the filament must also contribute to a lack of recognition by TLR5 (Holzapfel, Bonhomme et al. 2020). Many of these Leptospira glycosylation sites are conserved amongst spirochete FlaBs (Kurniyati, Kelly et al. 2017), suggesting that similar modifications may be observed in the FlaB core of other species, providing roles in sheath-protein binding, and evasion from the host immune response.

\section{Role of FlaB isoforms}

Based on the glycosylation patterns and mass spectrometry analyses, we identified the core of the $L$. biflexa fcpA- filament as being composed primarily of FlaB4. Previous studies of wild-type $L$. 
interrogans showed a vast discrepancy in the number of copies of each FlaB isoform, ranging from $\sim 12,000$ copies per cell of FlaB1 to 300 copies per cell of FlaB3 (Beck, Malmstrom et al. 2009). Therefore, it was surprising to identify FlaB4, and not FlaB1, as the major component of all 11 protofilaments of our $f c p A^{-}$structure, even though both isoforms are equally abundant in our $f c p A^{-}$ filament samples (Table S1). On the one hand this could reflect genuine differences in protein stoichiometries between $L$. interrogans and L. biflexa flagella. On the other hand, perhaps other regions of the core (with no FlaA2/FlaAP bound) are more heterogeneous in terms of FlaB composition. As FlaB4-specific glycosylation sites and loops appear critical to the interaction between the sheath and the core, it is likely that this isoform is required for FlaA2 and FlaAP binding. Whether this isoform is dominant in the wild-type $L$. biflexa structure remains unknown, as the resolution of the core in our current wild-type structure unfortunately barred a detailed analysis.

In the $L$. interrogans flaA2 filament, however, the major isoform appeared to be FlaB1. The sheath-glycan interactions and most of the sheath-core protein-protein contacts are not FlaB1-specific, raising the possibility that the Fcp sheath factors may have the ability to bind to various FlaB isoforms. This could explain the ability of these coiling factors to assemble symmetrically in these mutant filaments, as these Fcp sheath factors would still be able to bind even if the inner curvature of the core consisted of a different FlaB isoform.

\section{Role(s) of additional, undefined sheath proteins}

While mass spectrometry analyses of the purified flagella indicate that FlaA1 is present in the $f c p A^{-}$filaments (Table S1), we were unable to identify it in our structure, suggesting that FlaA1 is not as stably bound to the core. A previous study of L. biflexa failed to detect FlaA1 in purified fcpA- filaments, though the sheath factor was present in the cell lysate (Sasaki, Kawamoto et al. 2018); this contrasts results from $L$. interrogans in which FlaA1 was indeed detected in purified fcpA- filaments (Wunder, Figueira et al. 2016). The presence of additional decorated protofilaments in a minority of our analyzed $f c p A^{-}$filaments may reflect the presence of this and/or other sheath components.

The wild-type structure appears to contain additional sheath factors, with densities that do not correspond to known components -FlaA2, FlaAP, FcpA, FcpB, or the FlaB core (Fig. 6A). While one of these densities is likely FlaA1, the fitting of a predicted model was not conclusive, due to limited local resolution of the map segments, and/or actual model inaccuracies. Additional proteins are anticipated, which would correspond to novel flagellar protein species, future studies shall shed light into their identities, precise location and roles in endoflagellar filament assembly. Altogether, these additional factors are also located near the inner curvature of the filament, adjacent to the FcpA coiling factors. These factors may therefore also play a role in 'templating' the sheath, likely stabilizing the wild-type asymmetric sheath arrangement or otherwise facilitating its formation.

\section{Conclusion}

The Leptospira flagellum is a complex assembly, composed of multiple different types of proteins- a glycosylated FlaB core, FlaA 'templating' factors, and Fcp 'coiling' factors. The simultaneous localization of the FlaA sheath factors to the inner curvature and the Fcp factors to the outer curvature, results in a functional, supercoiled filament; without both types of sheath proteins, the filament remains 
relaxed and is unable to bend into the tight coils that are associated with motility. Continued investigations of spirochete flagellar architecture will help to inform how these remarkable filaments play such a vital role in the unique movement of this important bacterial phylum.

\section{Methods}

\section{Strains and culturing of Leptospira}

Cultures of Leptospira biflexa serovar Patoc strain Patoc I (Paris) wild-type and $f c p A^{-}$mutant samples (Wunder, Slamti et al. 2018), as well as L. interrogans serovar Manilae flaA2- (Lambert, Picardeau et al. 2012), were grown in Ellinghausen-McCullough-Johnson-Harris (EMJH) liquid medium at $30^{\circ} \mathrm{C}$ (Wunder, Figueira et al. 2016).

\section{Flagella purification}

Purification of $L$. biflexa $f c p A^{-}$and $L$. interrogans flaA2- flagella was performed as previously described (Miller, Miller et al. 2016). Briefly, $500 \mathrm{~mL}^{\circ}$ of a cpA $^{-}$or flaA2 culture was harvested and centrifuged at $8000 \mathrm{xg}$ for 15 minutes, and the pellet was washed with cold PBS and re-centrifuged. The pellet was resuspended in $30 \mathrm{~mL}$ Tris buffer $(150 \mathrm{mM}$ Tris (hydroxymethyl amino methane, $\mathrm{pH} 6.8$, with $0.9 \%$ sodium chloride) and centrifuged as before. The pellet was then resuspended in $15 \mathrm{~mL}$ of Tris buffer, and stirred at $4^{\circ} \mathrm{C}$ for 10 minutes, before $1.5 \mathrm{~mL}$ of $20 \%$ TritonX-100 was added to the sample. After stirring at room temperature for 1 hour, the sample was centrifuged at 15,000xg for 45 minutes, and the pellet was resuspended in $15 \mathrm{~mL}$ Tris buffer. 1000 units of mutanolysin was added dropwise to the sample, which was stirred at room temperature for 1 hour, and then overnight at $4^{\circ} \mathrm{C}$. The sample was then centrifuged at $8,000 \times \mathrm{xg}$ for 30 minutes. The pellet was discarded, and $2.2 \mathrm{~mL}$ of ammonium sulfate was added to the supernatant (for a final ammonium sulfate concentration of $12.6 \%$ ); this was stirred at $4^{\circ} \mathrm{C}$ for 20 minutes. This sample was then centrifuged at 120,000xg for 2 hours. The resulting pellet was resuspended in water, and the centrifugation was repeated. The final pellet was resuspended in $600 \mu \mathrm{L}$ water. The sample was then analyzed with SDS-PAGE and Western blots to ensure that the expected flagellar proteins were present.

L. biflexa WT flagella were purified as previously described (Wunder, Figueira et al. 2016). Briefly, $500 \mathrm{~mL}$ of a L. biflexa WT culture were harvested and centrifuged at $8000 \times \mathrm{xg}$ for 15 minutes. The pellet was washed with cold PBS and re-centrifuged. The pellet was then suspended in $30 \mathrm{~mL} 0.15 \mathrm{M}$ Tris. $\mathrm{HCl}$ pH8.0, 0.5M sucrose and centrifuged as before. The pellet was then resuspended in $30 \mathrm{~mL}$ of Tris-sucrose buffer $(0.5 \mathrm{M}$ sucrose, Tris $150 \mathrm{mM}, 50 \mathrm{mM} \mathrm{NaCl})$, stirred at $4^{\circ} \mathrm{C}$ for 10 minutes, and then 3 $\mathrm{mL} 10 \%$ TritonX-100 were added. After stirring at room temperature for 30 minutes, $0.1 \mathrm{mg} / \mathrm{mL}$ (final concentration) hen egg-white lysozyme, $0.005 \mathrm{mg} / \mathrm{mL}$ DNAse, $0.01 \mathrm{mg} / \mathrm{mL}$ RNAse and $2 \mathrm{mM} \mathrm{MgCl}$ were sequentially added dropwise, further stirring at room temperature for 2 hours. The samples were then stirred 10 minutes with $2 \mathrm{mM} \mathrm{MgSO}_{4}$, and then 10 minutes with $2 \mathrm{mM}$ EDTA pH8.0, then centrifuged at $17000 x$ for 15 minutes. The pellet was discarded, and $4 \mathrm{~mL}$ of $20 \%$ PEG8000, $1 \mathrm{M} \mathrm{NaCl}$ were added to the supernatant and incubated for $30 \mathrm{~min}$ at $4^{\circ} \mathrm{C}$. This sample was centrifuged at $27000 \mathrm{xg}$ for $30 \mathrm{~min}$, and the pellet resuspended in $4 \mathrm{~mL} 150 \mathrm{mM}$ Tris pH8.0, $50 \mathrm{mM} \mathrm{NaCl}$, and slowly stirred at $4^{\circ} \mathrm{C}$. Flagellar filaments were recovered by ultracentrifugation at $80000 \mathrm{xg}$ for 45 minutes, this final pellet was resuspended in $500 \mu \mathrm{L}$ of $150 \mathrm{mM}$ Tris $\mathrm{pH} 8.0,50 \mathrm{mM} \mathrm{NaCl}$. 


\section{Cryo-EM sample preparation}

3-4 $\mu \mathrm{L}$ of purified L. biflexa fcpA- flagella samples were applied to Quantifoil R1.2/1.3 Cu 300 mesh grids (Ted Pella, Inc., Redding, CA), 3-4 $\mu$ L of purified $L$. interrogans flaA2 flagella samples were applied to Quantifoil R1.2/1.3 Cu200 mesh grids (Ted Pella, Inc., Redding, CA), and $2.5 \mu \mathrm{L}$ of L. biflexa wild-type purified flagella were applied to Quantifoil 2.3/1.3 Cu300 mesh grids (Ted Pella, Inc., Redding, CA) . All grids were plasma discharged in a Gatan Model 950 Solarus Advanced Plasma System with $\mathrm{H} 2 / \mathrm{O} 2$ for either 30 seconds ( $f c p A^{-}$and flaA2) or for 20 seconds (wild-type). The grids were incubated for 1 minute, and then plunge frozen with a Vitrobot Mark IV, with a blotting time of 6 seconds and a blotting force of 2 , all at $18-22^{\circ} \mathrm{C}$ and $100 \%$ humidity.

\section{Data Collection}

Initial micrographs of $f c p A^{-}$filaments were collected through the program SerialEM (Mastronarde 2005) on the 200kV Thermo Scientific Glacios containing a K2 detector. Images were collected with a pixel size of $0.896 \AA$.

Wild-type, flaA2, and additional $f c p A^{-}$micrographs were collected on the $300 \mathrm{kV}$ Titan Krios microscope, containing a $\mathrm{K} 3$ detector, through the program SerialEM. $f c p A^{-}$and flaA2 images were collected with super-resolution pixel size of $0.534 \AA$ and a magnification of $81000 x$. A magnification of $130000 x$ was used for the wild-type sample, with a super-resolution pixel size of $0.525 \AA$. A defocus between $-1.5 \mu \mathrm{m}$ to $-3.2 \mu \mathrm{m}$ was used for the $f c p A^{-}$samples, and a defocus of $-1.5--2.6 \mu \mathrm{m}$ was used for the flaA2 samples, and a defocus of $-3.0 \mu \mathrm{m}$ was used for the wild-type sample. A total dose of $60 \mathrm{e}$ $/ \AA^{2}$ was used for both $f c p A^{-}$and flaA2 samples, and a total dose of $54 \mathrm{e}^{-} / \AA^{2}$ was used for the wild-type sample. $11906 f c p A^{-}$micrographs were collected; for 4994 of those micrographs, image shift was used to take images at four holes per stage position. 2465 flaA2 micrographs were obtained, and 718 wild-type micrographs were collected (197 at $0^{\circ}$ tilt, and 521 at $45^{\circ}$ tilt). For all micrographs, only one image was taken per hole.

\section{RELION reconstruction of the fcpA- filaments} Initial Glacios reconstruction

An initial helically symmetric model was generated from 802 micrographs collected on the Glacios. MotionCor2 (Zheng, Palovcak et al. 2017) was used for motion correction, Gctf (Zhang 2016) in Relion 3.0 (Zivanov, Nakane et al. 2018) was used for CTF correction. Manual selection of filaments was performed in EMAN (Ludtke, Baldwin et al. 1999), resulting in 74,369 image segments. 2D classification was performed to separate sheathed and bare filaments; 26,552 image segments (corresponding to filaments without a visible sheath) were selected for further reconstruction of the core. A $30 \AA$ low-pass filter of a Bacillus subtilis flagellar filament (EMDB-8852) was used as an initial reference (Wang, Burrage et al. 2017) for helical reconstruction. The following helical parameters were used: a $0.5^{\circ}$ local searches were used, 11 asymmetrical units, an initial helical rise of $4.72 \AA$ (with a $0.2 \AA$ search between $4.42 \AA$ and $5.02 \AA$ ), an initial helical twist of $65.3^{\circ}$ (with a $0.2^{\circ}$ search between $62.3^{\circ}$ and $68.3^{\circ}$ ), a central $Z$ length of $30 \%$, a range factor of local averaging of 2 , a psi angular search range of $10^{\circ}$, a tilt angular search range of $15^{\circ}$, an outer tube diameter of $280 \AA$, an initial angular sampling of $0.9^{\circ}$, and using fixed tilt-prior angles.

$\underline{\text { Initial Krios reconstruction }}$ 
11906 micrographs were collected on the Krios. The initial 4461 micrographs were also processed with Relion 3.0 (Zivanov, Nakane et al. 2018). Motion correction and CTF correction were performed with MotionCor2 (Zheng, Palovcak et al. 2017) and Gctf (Zhang 2016), respectively. Filament selection was performed with crYOLO (Wagner and Raunser 2020), using filaments manually selected from 20 random micrographs with EMAN2 (Tang, Peng et al. 2007) for training, and was run with a box size of 320 pixels and a box distance of 56 pixels. This procedure yielded 807,689 image segments. Misselected particles were removed with 2D classification, yielding 803,924 image segments. The pf_smooth program (Debs, Cha et al. 2020) was used to remove discontinuities from the filament trajectories, with the following filament parameters: a rise per subunit of $4.72 \AA$, a twist per subunit of $65.356^{\circ}, 11$ protofilaments, a window size of 7 , a fit order of 2 , a minimum filament length of 10 , a direction tolerance of 20 , and a phi, theta, psi, distance, and twist tolerance of 10 . After four iterations of smoothing, 430,755 image segments remained (53.3\% of the crYOLO-selected image segments).

3D helical refinement was performed on these smoothed image segments. A $10 \AA$ low-pass filtered map of the FlaB core structure from the Glacios data as a reference, and the same helical parameters as the Glacios refinement were used. To account for the asymmetry of the sheathed filament, the resulting star file was then expanded 11-fold with relion_particle_symmetry_expand (utilizing the helix function, with 11 protofilaments, a twist of $65.4^{\circ}$, and a rise of $4.72 \AA$ ), resulting in 4,738,305 image segments.

Due to the asymmetry and heterogeneity present in the sample, additional image analysis steps were utilized to obtain meaningful 3D reconstructions (Mentes, Huehn et al. 2018). Particle subtraction was performed prior to focused 3D classification targeting the sheath. A cylindrical mask, $90 \AA$ in diameter and along the entire length of the reconstructed filament, was used for particle subtraction. An initial 3D classification was performed on a random subset of $\sim 70,000$ subtracted particles, using the following parameters: a $15 \AA$ filtered copy of the helical refinement as a reference, the same mask that was used in the subtraction, 5 classes, a regularization parameter of 100 , no image alignment, and no helical reconstruction. The classification converged by 27 iterations, and resulted in a sheathed class (9,942 particles, or $14.2 \%$ of asymmetric subunits), three bare classes (totaling 48,371 particles, or $69.0 \%$ of asymmetric subunits), and a class possibly corresponding to a part of the sheath that was cut off by the mask $(11,823$ particles, or $16.9 \%$ of asymmetric subunits). These classes were then used as a seed for the subsequent 3D classification on the full $\sim 4$ million particle dataset, utilizing the same parameters for classification. This run converged after 16 iterations, and resulted in one decorated sheath class (416,720 particles, or $8.8 \%$ of asymmetric subunits), three bare classes (totaling 4,011,067 particles, or $84.7 \%$ of asymmetric subunits), and a final class that may represent part of the sheath that was cut off by the mask (310,518 particles, or $6.6 \%$ of asymmetric subunits).

\section{Final Krios reconstruction}

All 11906 micrographs were then processed using Relion3.1 (Zivanov, Nakane et al. 2020). Motion correction, defocus estimation, and particle selection were performed as before, resulting in $1,632,891$ total image segments. Roughly half of these image segments did not have converged alignment parameters, with numerous discontinuities in the filament trajectories, hindering initial efforts at helical processing. An initial alignment was therefore generated using one round of Relion refinement (Class3D) with a fine-grained, exhaustive search. The following parameters were used: a regularization parameter of 4 , an angular sampling interval of $1.8^{\circ}$, an offset search range of 20 pixels with a 1 pixel search step, with no local angular searches performed, and using helical reconstruction 
with a tube outer diameter of $170 \AA$, a tilt search range of $15^{\circ}$, a psi search range of $20^{\circ}$, with fixed tiltpriors, and without applying helical symmetry. The reference for this step was the sheathed class from the original 4461-micrograph analysis. Utilization of an $80 \AA$ high-pass filtered reference improved the ability to track continuously along filaments, possibly by de-emphasizing sheath features that could interfere with the alignment. After this classification, $p f$ smooth was applied using the same parameters as before, resulting in 1,538,897 image segments. 2D classification was used to discard $\sim 1000$ misselected image segments, and Relion 3D helical refinement was performed on the remaining 1,538,130 image segments, using the same parameters as the initial Krios reconstruction. Particle expansion (yielding 16,919,430 image segments) and subtraction were performed as before. Asymmetric 3D classification was then performed, seeded by the classes identified in the 4461-micrograph analysis, with convergence achieved after 19 iterations. This resulted in a decorated sheath class $(816,888$ particles, or $4.8 \%$ of asymmetric subunits), three bare classes $(14,276,994$ particles for $84.4 \%$ total of asymmetric subunits), and a final class that may represent part of the sheath cut-off by the mask $(1,825,548$ particles, or $10.8 \%$ of asymmetric subunits). An additional round of focused classification was performed on the sheathed class, utilizing a smaller cylindrical mask focused on the FlaA2 density. This converged after 38 iterations, resulting in one class with strong density $(300,616$ particles, corresponding to $36.8 \%$ of the particles in the second classification and $1.8 \%$ of the overall particles), one class with moderate density $(40,595$ particles, corresponding to $5 \%$ of the particles in the second classification and $0.2 \%$ of particles overall), and two classes with poorly defined density $(475,677$ particles corresponding to $58.3 \%$ of the second classification and $2.8 \%$ of all the particles).

\section{Reconstruction of flaA2- filaments}

The flaA2 micrographs were initially processed with Relion3.1 (Zivanov, Nakane et al. 2020), with motion correction and CTF correction performed by MotionCor (Zheng, Palovcak et al. 2017) and gctf (Zhang 2016), respectively. Filaments were selected with crYOLO (Wagner, Lusnig et al. 2020); the program was trained using filaments manually selected from 20 micrographs with EMAN2 (Tang, Peng et al. 2007). All flagellar filaments (including those skinny and thick, straight and curved) were selected; resulting in 251,215 particles from 9670 filaments. The crYOLO filament selections were manually separated into a straight/thick, intermediate/curved, or skinny/curved group. The straight, thick filaments ( $24 \mathrm{~nm}$ in diameter) were used for all subsequent analysis, consisting of 36,151 image segments. These filaments were extracted in Relion with a box size of 384, and an initial 3D volume was generated using 11-fold symmetry, using a low-passed volume of the $f c p A^{-}$core model as a reference.

All subsequent refinement steps were carried out in cryoSPARC (Punjani, Rubinstein et al. 2017). To ensure that the majority of the filaments were thicker and straight, one round of 2D classification was performed; no particles were discarded. Helical refinement was then performed, using a low-passed volume of the Relion-generated structure as an initial reference. For all steps, 11 -fold symmetry, with a twist of $65.3^{\circ}$ and a rise of $4.72 \AA$, was used. Global CTF refinement and local CTF refinement were performed, before an additional round of helical refinement, utilizing the same parameters as before. $A$ resolution of $2.9 \AA$ was reported for the final helical reconstruction.

\section{Reconstruction of wild-type filaments}

Gain referencing was performed in IMOD (Kremer, Mastronarde et al. 1996) using the program 'clip', and the particles to a pixel size of $2.19 \AA$ using the IMOD program 'newstack'. All further processing and refinement steps were carried out in cryoSPARC v.3.3.1 (Punjani, Rubinstein et al. 2017). The Patch Correction tool (Rubinstein and Brubaker 2015) was used for motion correction, followed by multi-frame patch CTF estimation; default parameters were used for each. The filament tracer tool (in 
template-free mode) was used for filament selection, using a $300 \AA$ diameter and a fractional separation distance of 0.173. 40,061 particles were extracted, with a box size of 192 pixels. Per-particle local motion correction was applied, and misselected particles were removed with 2D classification; this left 36,638 particles. Helical refinement was performed with the following parameters: an initial mask based on the tomographic wild-type structure (Gibson, Trajtenberg et al. 2020), automasking enabled, and using initial helical parameters of a $0^{\circ}$ twist, a $52 \AA$ shift, and a maximum initial tilt search of $\pm 20^{\circ}$. The resultant structure had a reported resolution of $5.44 \AA$. Local per-particle CTF correction was performed (using default parameters) before repeating helical refinement; this structure gave a reported resolution of $4.5 \AA$. Global CTF refinement (including beam tilt, spherical aberration, and higher order trefoil and tetrafoil terms) and local CTF refinement were repeated once, followed by an additional round of helical refinement. The resulting structure reported a resolution of $4.28 \AA$, roughly the Nyquist limit of the sample. Heterogeneous refinement was used, utilizing two classes based on sheath density. The class with a more complete sheath contained 26,788 particles. Additional rounds of helical and non-uniform refinement further improved the high-frequency signal.

\section{Mass Spectrometry}

Flagella purification for LC-MS/MS:

L. biflexa fcpA- were purified for mass spectrometry using the method described above for the wild-type samples. Protein concentration of the extracts were determined by SDS-PAGE and densitometry analysis using the LMW-SDS Marker Kit (GE Healthcare) as standard.

\section{Nano LC-MS/MS analysis and protein identification:}

Flagellar extracts were run on $12 \%$ acrylamide SDS polyacrylamide gels and processed as previously described (Rossello, Lima et al. 2017). Briefly, $20 \mu \mathrm{g}$ of proteins of each replicate were run until samples entered $1 \mathrm{~cm}$ into the resolving SDS-PAGE. After slicing, each band was destained and cysteine alkylation was performed in-gel by successive incubation with $10 \mathrm{mM}$ dithiothreitol for $1 \mathrm{~h}$ at $56^{\circ} \mathrm{C}$ and then $55 \mathrm{mM}$ iodoacetamide at room temperature for 45 minutes. In-gel proteolytic digestion was performed overnight at $37^{\circ} \mathrm{C}$ using sequencing-grade trypsin (Promega). The resulting peptides were extracted at room temperature with $50 \%$ acetonitrile/ $0.1 \%$ trifluoroacetic acid. Peptides were desalted using $\mathrm{C} 18$ microcolumns (ZipTip ${ }^{\circledR} \mathrm{C} 18$, Millipore), vacuum dried and resuspended in $20 \mu \mathrm{L}$ of $0.1 \%$ formic acid.

Three biological replicates of flagella purifications were analyzed using a nano-HPLC (UltiMate 3000, Thermo) coupled to a Q-Orbitrap mass spectrometer (Q Exactive Plus, Thermo). Tryptic peptides $(5 \mu \mathrm{g})$ were separated into a $75 \mu \mathrm{m} \times 50 \mathrm{~cm}$, PepMapTM RSLC C18 analytical column $(2 \mu \mathrm{m}$ particle size, $100 \AA$, Thermo) at a flow rate of $200 \mathrm{~nL} / \mathrm{min}$ using a 90 minutes gradient (from $1 \%$ to $35 \%$ of acetonitrile in $0.1 \%$ formic acid). Mass analysis was performed in a data-dependent mode (full scan followed by $\mathrm{MS} / \mathrm{MS}$ of the top $12 \mathrm{~m} / \mathrm{z}$ in each segment) using a dynamic exclusion list.

PatternLab for Proteomics (Version V, http://www.patternlabforproteomics.org/) was used for protein identifications (Carvalho, Lima et al. 2016). Briefly, raw data were searched against a target decoy database including Leptospira biflexa serovar Patoc_UP000001847 sequences downloaded from Uniprot (December, 2021) and 127 most common mass spectrometry contaminants. Search parameters were set as follows: enzyme: trypsin; enzyme specificity: full specific; oxidation of methionine as variable modification and carbamidomethylation as fixed modification; $35 \mathrm{ppm}$ of tolerance from the measured precursor $\mathrm{m} / \mathrm{z}$. Peptide spectrum matches were filtered using the Search Engine Processor (SEPro) using the following parameters: acceptable FDR: $1 \%$ at the protein level; a minimum of two 
peptides per protein and 10 ppm precursor mass tolerance. Patternlab for proteomics's Venn Diagram module was used to identify proteins present in all replicates. The mass spectrometry proteomics data have been deposited to the ProteomeXchange Consortium via the PRIDE (Perez-Riverol, Csordas et al. 2019) partner repository with the dataset identifier PXD030741.

\section{Model building}

AlphaFold2 (Jumper, Evans et al. 2021) was used to generate structural predictions for the $L$. biflexa proteins FlaB1, FlaB2, FlaB3, FlaB4, FlaA2, and FlaA1. For the $f c p A^{-}$core region, each FlaB AlphaFold2 model was manually fit into the density in ChimeraX (Goddard, Huang et al. 2018), and then Isolde (Croll 2018) was used to individually improve the fit of each isoform (Table S2). We identified FlaB4 as the presumptive isoform present in the sample (based on the glycan fingerprint), and used Isolde to individually fit the FlaB4 monomer into each of the 11 unique protofilaments.

In the $f c p A^{-}$sheath region, the AlphaFold 2 FlaA2 model was manually aligned to the beta-sheet density in ChimeraX, and then Isolde was used to fit the sequence into the density. Bulky side chains were fit into lobes in the corresponding density, helping to confirm the sequence registration and helping to confirm the identity of the density as FlaA2.

Structural predictions of uncharacterized proteins identified in abundance in our sample through mass spectrometry were also obtained with AlphaFold2. One protein, the product of LEPBI_10551, was predicted to form an alpha-helical bundle, similar to the observed helical sheath density associated to FlaA2. This model was manually aligned into our density with ChimeraX (Goddard, Huang et al. 2018), and then fit into the density with Isolde (Croll 2018).

AlphaFold2 (Jumper, Evans et al. 2021) was also used to generate structural predictions of the $L$. interrogans proteins FlaB1, FlaB2, FlaB3, and FlaB4. As with the L. biflexa samples, each FlaB isoform was individually fit into the density with Isolde (Croll 2018). The fit of several FlaB1-specific bulky side chains and the lack of density for specific bulky residues in the other isoforms suggested a majority FlaB1 population. As this flagellum (flaA2-) is symmetric, FlaB1 monomers were placed into each FlaB protofilament without additional Isolde refinements.

FcpA and FcpB have been crystallized in L. biflexa and L. interrogans, respectively (San Martin, Mechaly et al. 2017, Gibson, Trajtenberg et al. 2020). AlphaFold2-predicted structures of these proteins in L. interrogans closely resembled the crystal structures, and were used as an initial fit into the flaA2 density. Isolde was then used to further refine the models.

The Isolde-modeled structures of the L. biflexa FlaB4 core, FlaA2, and FlaAP, as well as the $L$. interrogans FcpA and $\mathrm{FcpB}$, were fit into the wild-type density using ChimeraX. Refinement of these models in the wild-type structure was not performed.

\section{Docking of the FlaA2 and FlaAP models into the wild-type density}

The program Situs (Wriggers 2012, Kovacs, Galkin et al. 2018) was employed to dock our FlaA2 and FlaAP models into the wild-type density. The inner-curvature density (which remained unidentified in the tomographic wild-type structure) was used for the docking. A model consisting of one FlaA2 and one FlaAP monomer was used for the docking, which was performed with the program colores, using the inner core wild-type map, an angular sampling of $10^{\circ}$, and an anisotropy factor of 4 . Manual docking of these sheath factors in the wild-type density and model, using a model consisting of one core repeat 
(11 protofilaments), one FlaA2 monomer, and one FlaAP monomer, was consistent with the Situs fittings.

\section{Acknowledgments}

This work was supported by National Institutes of Health grants R01 GM110530 (to C. V. S.), T32GM8283 (to M.R.B) and U01 Al 088752, R01 TW009504, R01 Al052473, R0 Al121207 (to A. K.). ; Agencia Nacional de Investigacion e Innovacion grant FCE_3_2016_1_126797 and Agence Nationale de la Recherche grant ANR-18-CE15-0027-01 (to A. B). Cryo-EM data was collected at the Yale CryoEM Resource that is funded in part by the NIH grant 1S100D023603-01A1. We also thank the Center for Cellular and Molecular Imaging Electron Microscopy Facility at the Yale Medical School for assistance with this work and thank the Yale Center for Research Computing and the Yale High Performance Computing facility for guidance and use of the research computing infrastructure. 


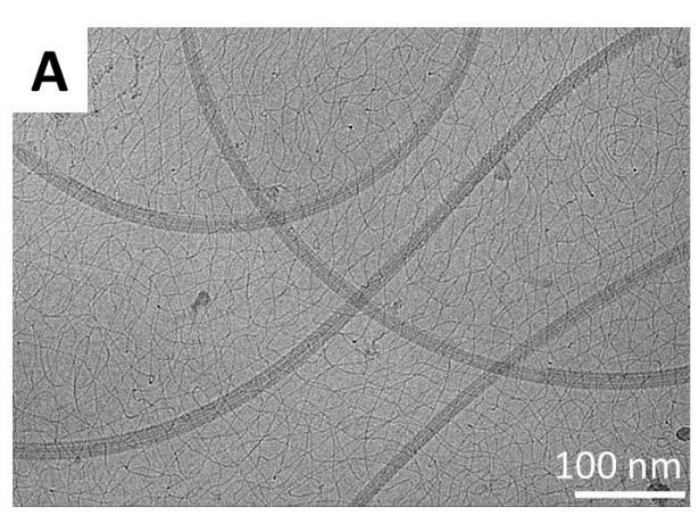

C

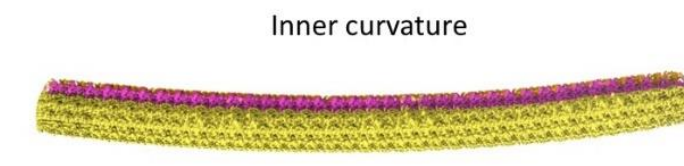

Proximal

Outer curvature

E Inner curvature

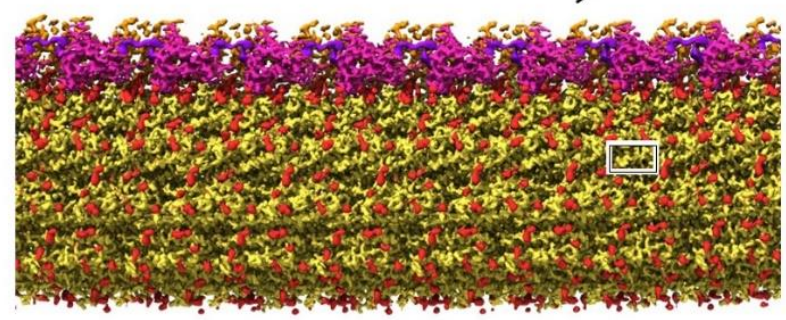

Proximal

Outer curvature

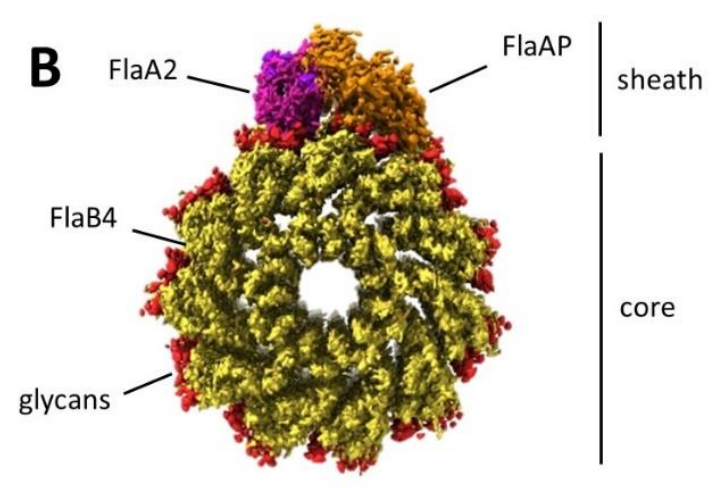

D
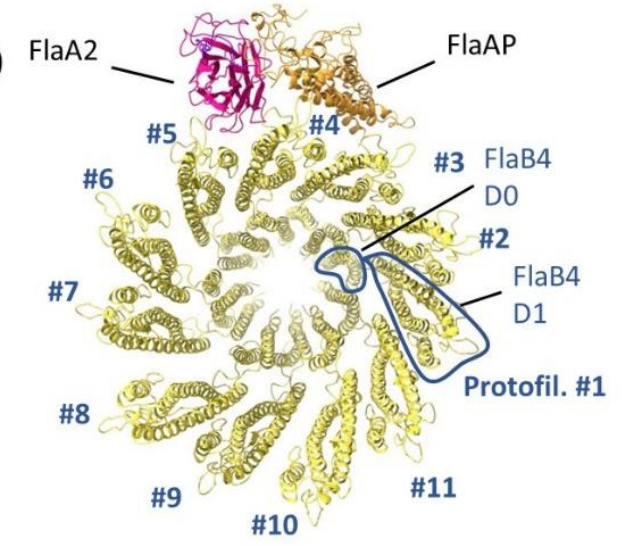

$\mathbf{F}$

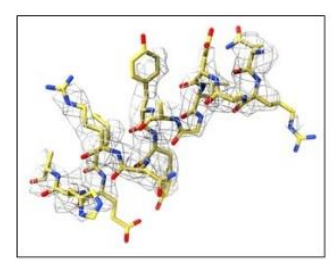

Figure 1. FlaA2 co-localizes to the filament inner curvature in a single row together with FlaAP, a previously uncharacterized protein. In this and the following figures, 'proximal' and 'distal' labels indicate the direction of the polar filament with respect to the flagellar motor. A, Representative micrograph, showing the heterogeneity and slight curvature of the $f c p A^{-}$filaments. Scale bar is $100 \mathrm{~nm}$. B, A 3D isosurface rendering of a flagellar filament decorated with FlaA2 and FlaAP. Density for the FlaB core is colored yellow, density identified as bound FlaA2 molecules is colored pink, and density corresponding to FlaAP is colored orange. Putative glycosylation site densities are colored red, and the C-terminal 'tentacle' of FlaA2 is colored purple. C, A lower magnification view of an extended filament generated from the reconstruction in A, revealing supercoiling. Estimated supercoil pitch and diameter values are $2.01 \mu \mathrm{m}$ and $0.43 \mu \mathrm{m}$ respectively. D, Models of the FlaB4 core and FlaA2 and FlaAP sheath proteins, using the same coloring as in $\mathbf{B}$. The initial models were generated with AlphaFold 2 , and were fit into the density using Isolde. E, Side view of the reconstructed $f c p A^{-}$filament, as in B. F, A zoomed-in view of the box in $\mathbf{E}$, showing electron density corresponding to an alpha-helical segment of FlaB4. 
bioRxiv preprint doi: https://doi org/10 1101/2022 03.03 482903; this version posted March 3, 2022. The copyright holder for this preprint (which was not certified by peer review) is the author/funder, who has granted bioRxiv a license to display the preprint in perpetuity. It is made available under aCC-BY 4.0 International license.
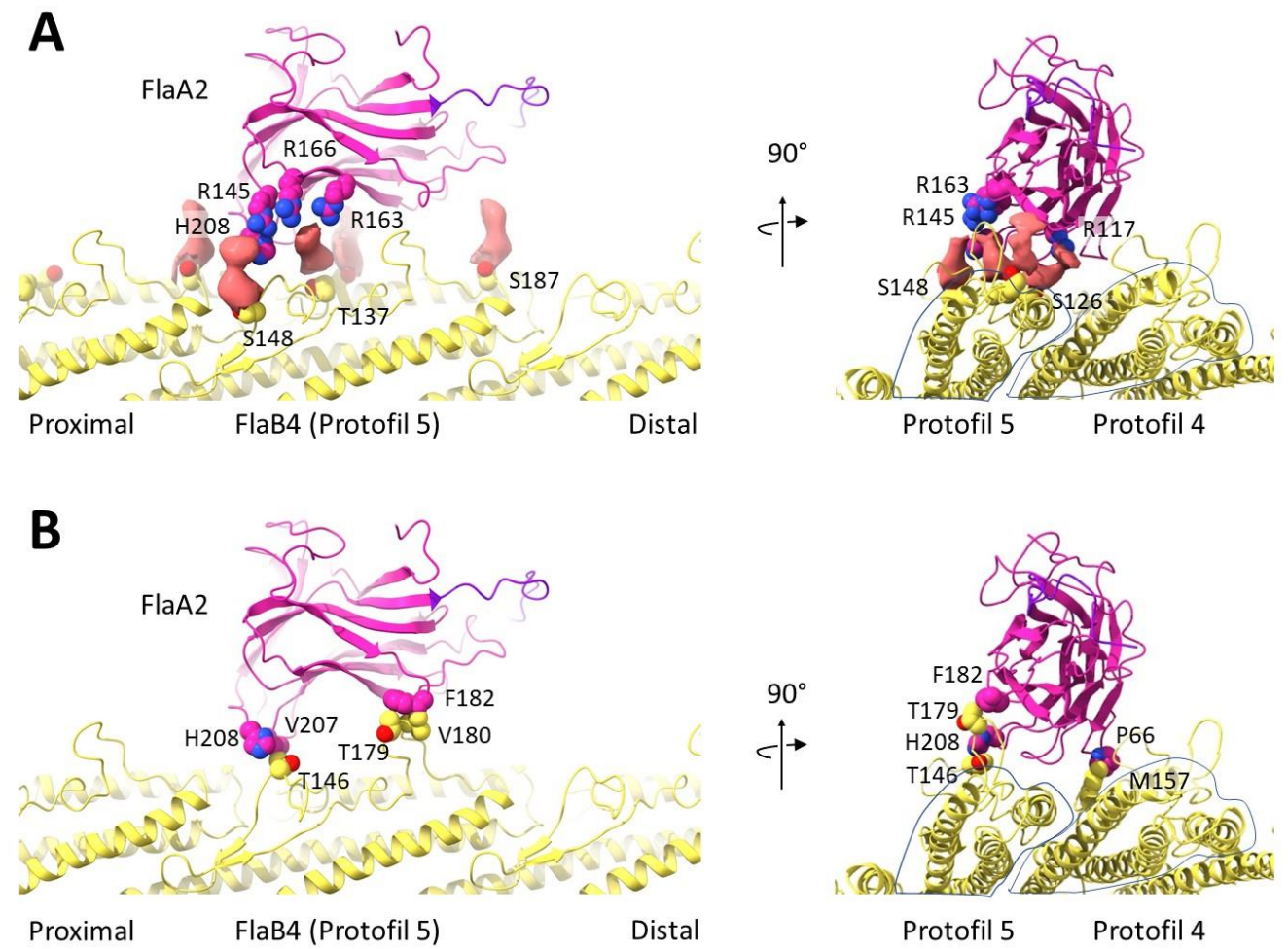

FlaB4 (Protofil 5)

Protofil $5 \quad$ Protofil 4
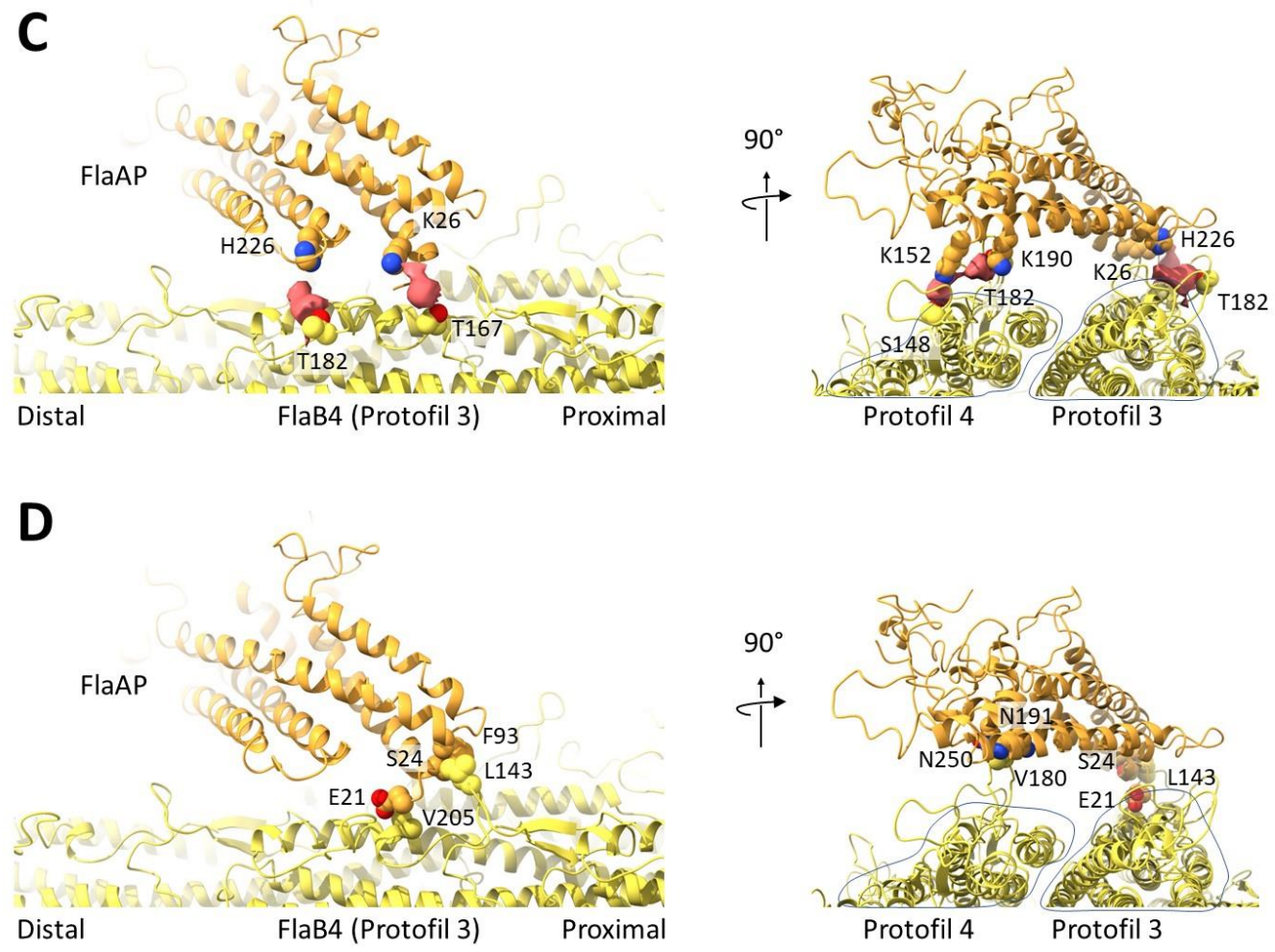
Figure 2. Core interactions of FlaA2 and FlaAP are primarily mediated by glycosylated FlaB4 side chains. A, Fit atomic model of FlaA2 highlighting probable sugar-binding sites. Proposed glycan moieties are not modeled, their segmented cryoEM densities are shown as solid pink surfaces. Left: The presumed glycan of $\mathrm{Ser}_{148}$ (FlaB4) interacts with $\mathrm{Arg}_{145}$ and $\mathrm{His}_{208}$ (FlaA2), and the presumed glycan on $\mathrm{Thr}_{137}$ (FlaB4) interacts with $\operatorname{Arg}_{163}$ and $\operatorname{Arg}_{166}$ (FlaA2). Right: End-on view of the filament shows that the glycans are all located along one protofilament (\#5). B, Protein-protein interactions between FlaA2 and the FlaB4 core. Left: $\mathrm{His}_{208}$ and $\mathrm{Val}_{207}$ (FlaA2) interact with Ser 146 (FlaB4), and Phe 182 (FlaA2) interacts with $\mathrm{Thr}_{179}$ and $\mathrm{Val}_{180}$ (FlaB4). End-on view, showing that these protein contacts bridge between two adjacent FlaB4 protofilaments (\#4 and \#5). C, Interactions between the FlaAP sheath protein and the glycans associated with the FlaB4 core. Left: The interaction between the presumed glycan on $\mathrm{Thr}_{182}$ (FlaB4) and $\mathrm{His}_{226}$ (FlaAP), and well as interactions between the presumed glycan of $\mathrm{Thr}_{167}$ (FlaB4) and Lys ${ }_{126}$ (FlaAP). Right: End-on view of the filament shows that these glycan interactions bridge between FlaB protofilaments, with two glycan interactions along both protofilaments \#3 and \#4. D, Protein-protein contacts between FlaAP and the FlaB4 core. Left: Ala ${ }_{172}$ (FlaB4) interacts with Glu ${ }_{21}$ (FlaAP), and Leu $_{143}$ (FlaB4) interacts with Phe $_{93}$ and $\mathrm{Ser}_{24}$ (FlaAP). Right: End-on view shows that the protein-protein contacts also help to bridge FlaAP between protofilaments \#3 and \#4. 

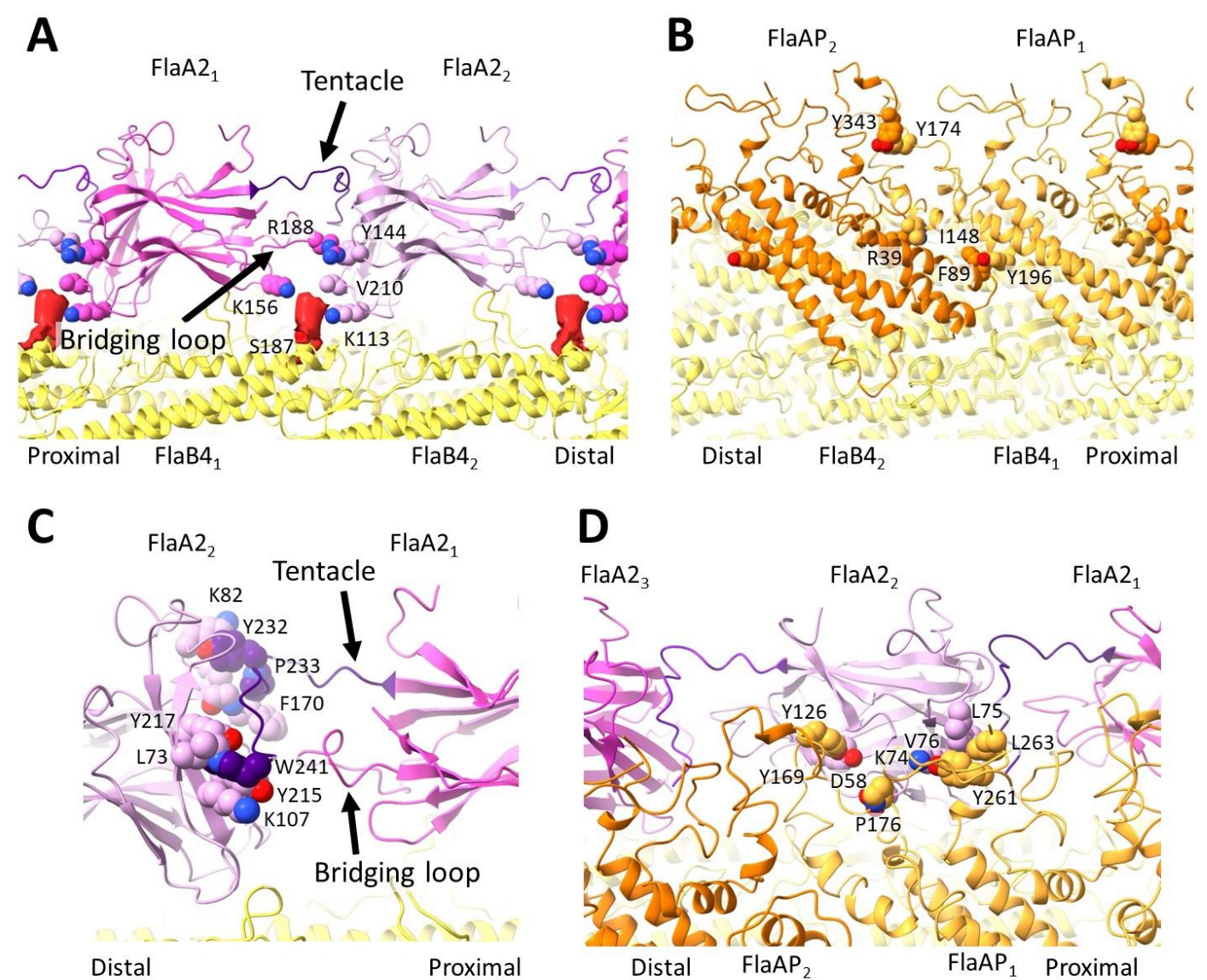

Figure 3. Core interactions of FlaA2 and FlaAP are primarily mediated by glycosylated FlaB4 side chains. A, Fit atomic model of FlaA2 reveals probable sugar binding sites. Proposed glycan moieties are not modeled, their segmented cryoEM densities are shown as solid red surfaces. Left: The glycan of Ser ${ }_{148}$ (FlaB4) interacts with $\mathrm{Arg}_{145}$ and $\mathrm{His}_{208}$ (FlaA2), and the glycan on $\mathrm{Thr}_{137}$ (FlaB4) interacts with $\mathrm{Arg}_{163}$ and $\operatorname{Arg}_{166}$ (FlaA2). Right: End-on view of the filament shows that the glycans are all located along one protofilament (protofilament \#5). B, Protein-protein interactions between FlaA2 and the FlaB4 core. Left: $\mathrm{His}_{208}$ and $\mathrm{Val}_{207}$ (FlaA2) interact with $\mathrm{Ser}_{146}$ (FlaB4), and Phe ${ }_{182}$ (FlaA2) interacts with $\mathrm{Thr}_{179}$ and $\mathrm{Val}_{180}$ (FlaB4). End-on view, showing that these protein contacts bridge between two adjacent FlaB4 protofilaments (protofilament \#4 and protofilament \#5). C, Interactions between the FlaAP sheath protein and the glycans associated with the FlaB4 core. Left: The interaction between the presumed glycan on $\mathrm{Thr}_{182}$ (FlaB4) and $\mathrm{His}_{226}$ (FlaAP), as well as interactions between the presumed glycan of Thr ${ }_{167}$ (FlaB4) and Lys 126 (FlaAP). Right: End-on view of the filament shows that these glycan interactions bridge between FlaB protofilaments, with two glycan interactions along both protofilaments \#3 and \#4. D, Protein-protein contacts between FlaAP and the FlaB4 core. Left: Ala ${ }_{172}$ (FlaB4) interacts with Glu ${ }_{21}$ (FlaAP), and Leu Le3 $_{143}$ (FlaB4) interacts with $\mathrm{Phe}_{93}$ and $\mathrm{Ser}_{24}$ (FlaAP). Right: End-on view shows that the protein-protein contacts also help to bridge FlaAP between protofilaments \#3 and \#4. 

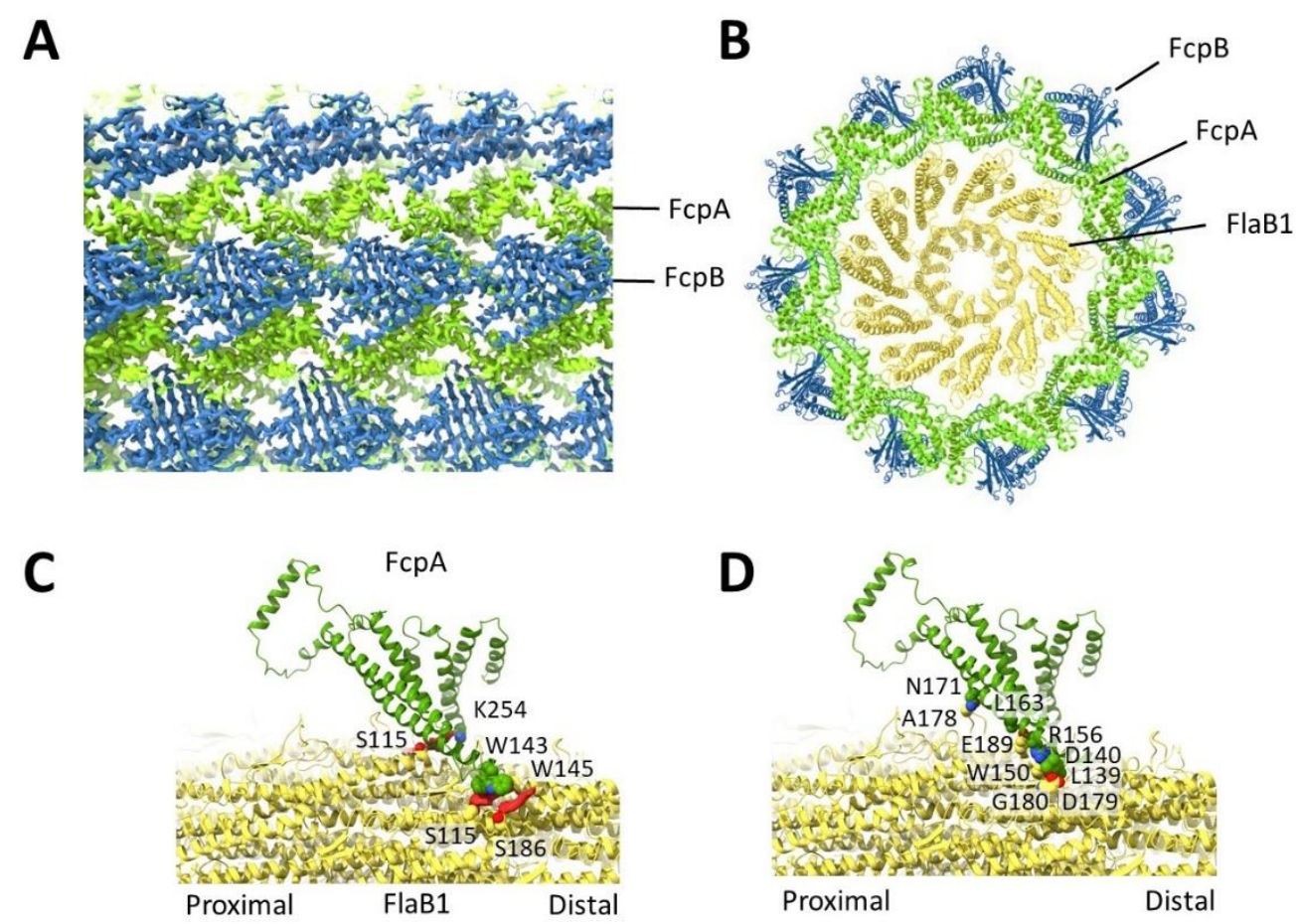

Figure 4. A lattice formed by FcpA and FcpB completely surrounds the core in flaA2 filaments, and is supported by interactions with glycosylated core side chains. A, flaA2 density map, highlighting the symmetric lattice of FcpA (green) and FcpB (blue) protomers. B, Atomic models of FlaB1 (yellow), FcpA (green), and $\mathrm{FcpB}$ (blue) after tracing the cryoEM maps. All three proteins conform to the 11-fold core helical symmetry, with FcpA and FcpB forming a continuous sheath that overlies the FlaB1 core. C, Each FcpA monomer contacts two FlaB1 protofilaments. Interactions are present between FcpA and presumably glycosylated residues of the FlaB1 core. Along one protofilament, $\operatorname{Trp}_{143}$ (FcpA) interacts with a glycan on $\operatorname{Ser}_{115}$ (FlaB1) and $\operatorname{Trp}_{145}$ (FcpA) interacts with a glycan on $\operatorname{Ser}_{186}$ (FlaB1). Lys 254 (FcpA) interacts with a glycan on Ser $_{115}$ of the adjacent FlaB1 protofilament. D, Protein-protein contacts between the FcpA sheath and the FlaB1 core occur at four locations, and mostly involve hydrophobic residues. 

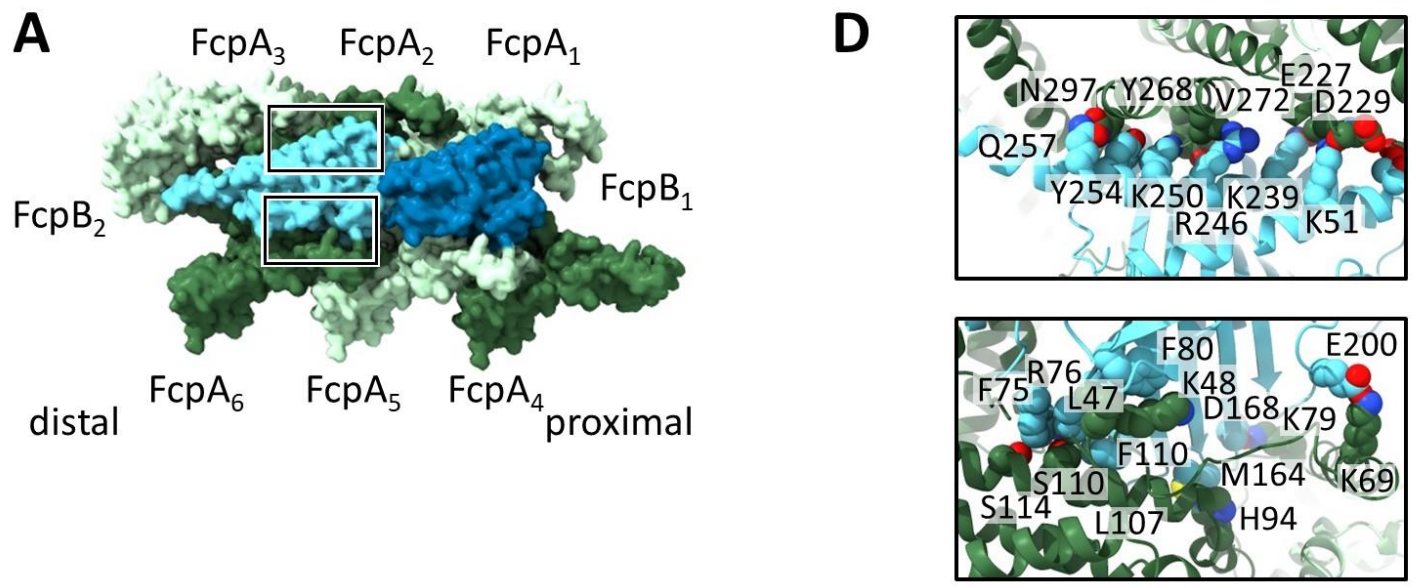

B

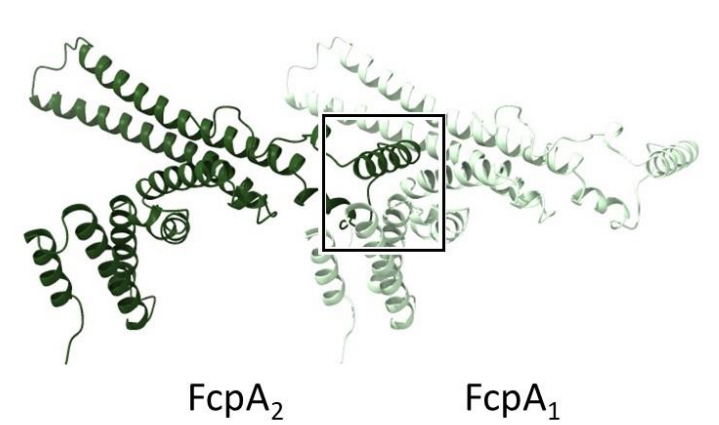

C

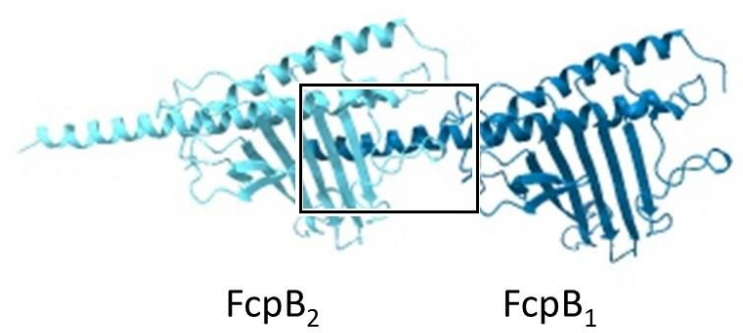

$\mathbf{E}$

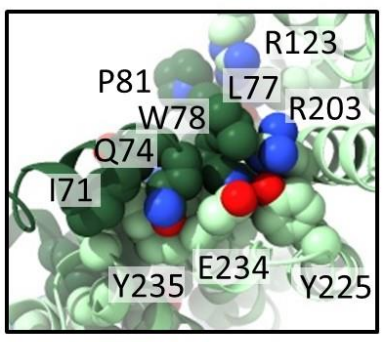

$\mathbf{F}$

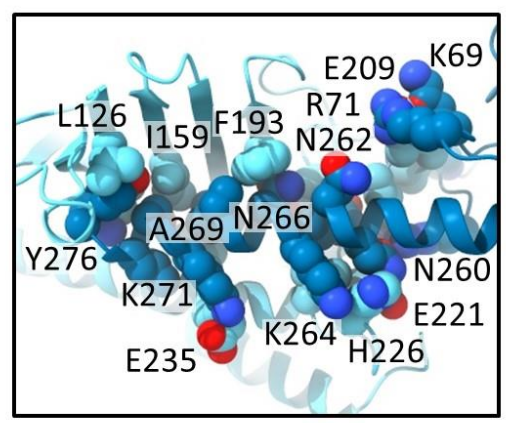

Figure 5. The $F c p A / F c p B$ lattice is characterized by extensive longitudinal interactions. A, FcpB overlies a lattice of FcpA, with each FcpB contacting five FcpA monomers. Contacts with two lattices are shown within the boxes; top box corresponding to the top panel in $\mathbf{D}$, and the bottom box corresponding to the bottom D panel. B, Extensive lateral FcpA interactions. The extended helix of one FcpA monomer contacts the proximal neighbor monomer. C, Lateral FcpB interactions occur through its long helix. D, $\mathrm{FcpA}$ and $\mathrm{FcpB}$ interactions. Top panel, contacts between $\mathrm{FcpB}_{2}$ and $\mathrm{FcpA}_{2}$. Bottom panel, contacts between $\mathrm{FcpB}_{2}$ and $\mathrm{FcpA}_{6}$. $\mathrm{E}$, Close-up view of the axial $\mathrm{FcpA}$ interactions, including hydrophobic interactions with $\operatorname{Trp}_{78}$ of $F c p A_{2}$ and a helix of $F c p A_{1}$. $F$, Lateral interactions of $F c p B$ involve contacts between the beta-sheet of one monomer and the helix of the proximal FcpB neighbor. 
A
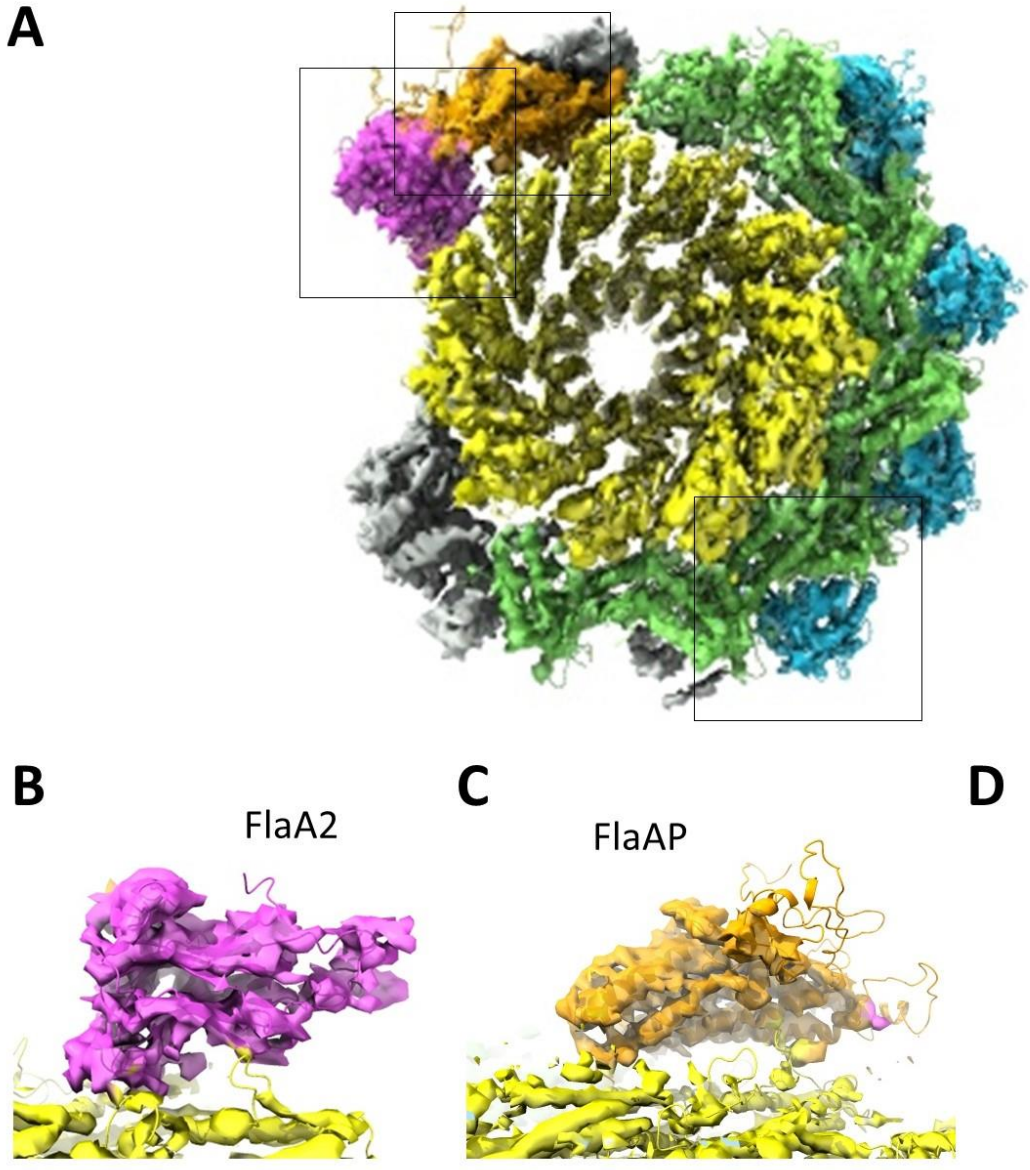

C

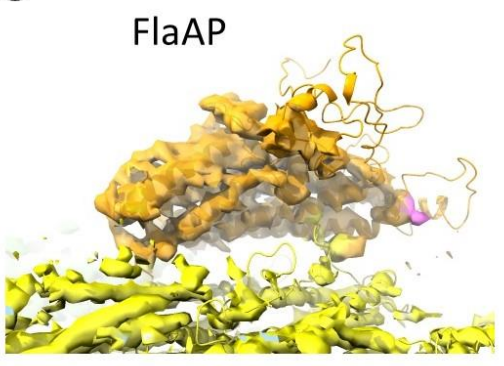

D
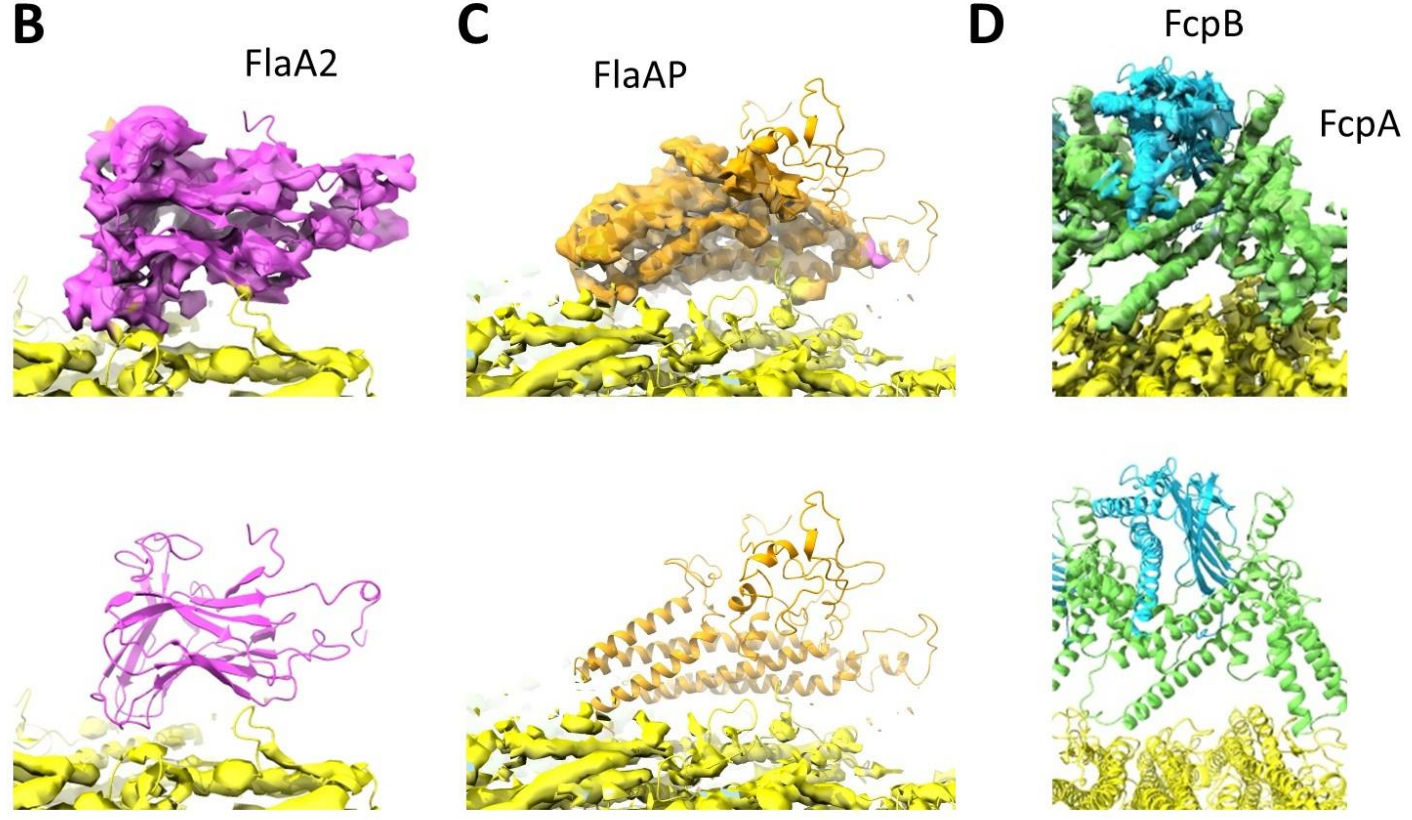

Figure 6. Lattice of FcpA and FcpB coiling proteins is interrupted by FlaA2/FlaAP in the wild-type Leptospira filament. A, Cross-sectional view of the wild-type filament structure. Density corresponding to the core is colored yellow, to FlaA2 is pink, to FlaAP is orange, to FcpA is green, and to FcpB is blue. Additional sheath density that does not correspond to these proteins is in gray. The three boxes highlight the regions featured in B, C, and D. B, Zoomed-in view of FlaA2 fit into the wild-type density. The top panel shows the density, the bottom shows the fit of the model within the density. FlaA2 is colored pink, and the FlaB model is colored yellow. C, FlaAP (orange) fit into the density, as in B. D, The fit of FcpA (green) and FcpB (blue) into the density, as in B. 


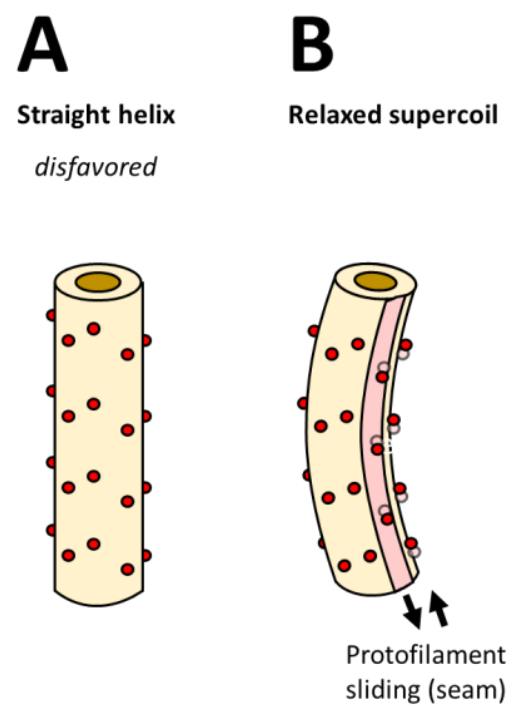

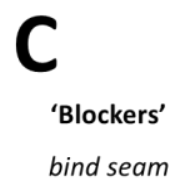

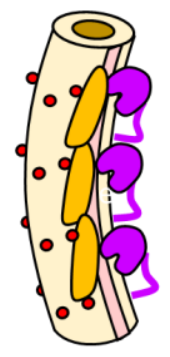

sliding (seam)
D

'Expanders'

bind outer curvature

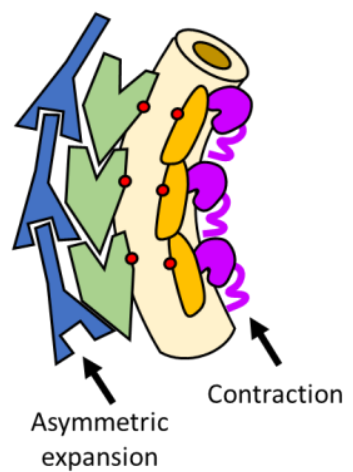

E

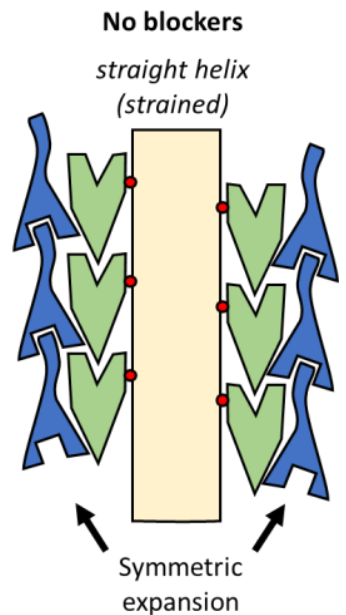

Figure 7. Model for joint cooperative activation of Leptospira flagellar filament function by multiple sheath proteins. A, Straight, helically symmetric core conformation; note that this is expected to be energetically disfavored. B, In the absence of sheath proteins, the FlaB core (yellow) assumes a relaxed supercoiled shape, like the 'normal' form observed in Salmonella and other bacteria. Red dots denote glycosylated core surface residues, following the filament helical lattice. A seam (pink) associated with the relaxed supercoil would feature sliding of several neighboring protofilaments, disturbing the helical lattice (A) on the inner curvature side of the filament. Specific targeting of FlaA2 (magenta) and FlaAP (orange) to the seam would then block binding of additional sheath proteins on the inner curvature (C). FcpA (green) and FcpB (blue) then function as 'expanders' upon binding the core (D), pushing against their longitudinal neighbors via specific binding interactions involving rigid secondary structure elements. Localization of FcpA/FcpB to the outer curvature (due to blocking by FlaA2, FlaAP and potentially other sheath proteins) thus promotes asymmetric lattice expansion on the outer curvature side of the filament. The fully assembled filament would thus adopt a tightly coiled form, enabling motility. Our wild-type structure indicates that several additional sheath proteins bind the inner curvature side of the filament (not shown). However, if FlaA2 and FlaAP are not present (as in the flaA2mutant), loss of blocking function enables the $\mathrm{FcpA} / \mathrm{FcpB}$ lattice to displace all other sheath components and fully envelop the FlaB core. The resulting filaments are helically symmetric and straight (E), and hence unable to support motility. 


\section{References}

Beatson, S. A., T. Minamino and M. J. Pallen (2006). "Variation in bacterial flagellins: from sequence to structure." Trends Microbiol 14(4): 151-155.

Beck, M., J. A. Malmstrom, V. Lange, A. Schmidt, E. W. Deutsch and R. Aebersold (2009). "Visual proteomics of the human pathogen Leptospira interrogans." Nat Methods 6(11): 817-823.

Berg, H. C. (2003). "The rotary motor of bacterial flagella." Annu Rev Biochem 72: 19-54.

Berg, H. C. and R. A. Anderson (1973). "Bacteria swim by rotating their flagellar filaments." Nature 245(5425): 380-382.

Blum, T. B., S. Filippidou, M. Fatton, P. Junier and J. P. Abrahams (2019). "The wild-type flagellar filament of the Firmicute Kurthia at 2.8 A resolution in vivo." Sci Rep 9(1): 14948.

Brahamsha, B. and E. P. Greenberg (1989). "Cloning and sequence analysis of flaA, a gene encoding a Spirochaeta aurantia flagellar filament surface antigen." J Bacteriol 171(3): 1692-1697.

Bromley, D. B. and N. W. Charon (1979). "Axial filament involvement in the motility of Leptospira interrogans." J Bacteriol 137(3): 1406-1412.

Calladine, C. R., B. F. Luisi and J. V. Pratap (2013). "A "mechanistic" explanation of the multiple helical forms adopted by bacterial flagellar filaments." J Mol Biol 425(5): 914-928.

Carvalho, P. C., D. B. Lima, F. V. Leprevost, M. D. Santos, J. S. Fischer, P. F. Aquino, J. J. Moresco, J. R. Yates, 3rd and V. C. Barbosa (2016). "Integrated analysis of shotgun proteomic data with PatternLab for proteomics 4.0." Nat Protoc 11(1): 102-117.

Croll, T. I. (2018). "ISOLDE: a physically realistic environment for model building into low-resolution electron-density maps." Acta Crystallogr D Struct Biol 74(Pt 6): 519-530.

Debs, G. E., M. Cha, X. Liu, A. R. Huehn and C. V. Sindelar (2020). "Dynamic and asymmetric fluctuations in the microtubule wall captured by high-resolution cryoelectron microscopy." Proc Natl Acad Sci U S A 117(29): 16976-16984.

Echazarreta, M. A., J. L. Kepple, L. H. Yen, Y. Chen and K. E. Klose (2018). "A Critical Region in the FlaA Flagellin Facilitates Filament Formation of the Vibrio cholerae Flagellum." J Bacteriol 200(15).

Evangelista, K. V. and J. Coburn (2010). "Leptospira as an emerging pathogen: a review of its biology, pathogenesis and host immune responses." Future Microbiol 5(9): 1413-1425.

Fontana, C., A. Lambert, N. Benaroudj, D. Gasparini, O. Gorgette, N. Cachet, N. Bomchil and M. Picardeau (2016). "Analysis of a Spontaneous Non-Motile and Avirulent Mutant Shows That FliM Is Required for Full Endoflagella Assembly in Leptospira interrogans." PLoS One 11(4): e0152916. Fouts, D. E., M. A. Matthias, H. Adhikarla, B. Adler, L. Amorim-Santos, D. E. Berg, D. Bulach, A. Buschiazzo, Y. F. Chang, R. L. Galloway, D. A. Haake, D. H. Haft, R. Hartskeerl, A. I. Ko, P. N. Levett, J. Matsunaga, A. E. Mechaly, J. M. Monk, A. L. Nascimento, K. E. Nelson, B. Palsson, S. J. Peacock, M. Picardeau, J. N. Ricaldi, J. Thaipandungpanit, E. A. Wunder, Jr., X. F. Yang, J. J. Zhang and J. M. Vinetz (2016). "What Makes a Bacterial Species Pathogenic?:Comparative Genomic Analysis of the Genus Leptospira." PLoS Negl Trop Dis 10(2): e0004403.

Fujii, M., S. Shibata and S. Aizawa (2008). "Polar, peritrichous, and lateral flagella belong to three distinguishable flagellar families." J Mol Biol 379(2): 273-283.

Ge, Y. and N. W. Charon (1997). "An unexpected flaA homolog is present and expressed in Borrelia burgdorferi." J Bacteriol 179(2): 552-556.

Gibson, K. H., F. Trajtenberg, E. A. Wunder, M. R. Brady, F. San Martin, A. Mechaly, Z. Shang, J. Liu, M. Picardeau, A. Ko, A. Buschiazzo and C. V. Sindelar (2020). "An asymmetric sheath controls flagellar supercoiling and motility in the leptospira spirochete." Elife 9. 
Goddard, T. D., C. C. Huang, E. C. Meng, E. F. Pettersen, G. S. Couch, J. H. Morris and T. E. Ferrin (2018). "UCSF ChimeraX: Meeting modern challenges in visualization and analysis." Protein Sci 27(1): 14-25. Goldstein, S. F. and N. W. Charon (1988). "Motility of the spirochete Leptospira." Cell Motil Cytoskeleton 9(2): 101-110.

Goldstein, S. F. and N. W. Charon (1990). "Multiple-exposure photographic analysis of a motile spirochete." Proc Natl Acad Sci U S A 87(13): 4895-4899.

Holzapfel, M., D. Bonhomme, J. Cagliero, F. Vernel-Pauillac, M. Fanton d'Andon, S. Bortolussi, L. Fiette, C. Goarant, E. A. Wunder, Jr., M. Picardeau, A. I. Ko, D. Werling, M. Matsui, I. G. Boneca and C. Werts (2020). "Escape of TLR5 Recognition by Leptospira spp.: A Rationale for Atypical Endoflagella." Front Immunol 11: 2007.

Jumper, J., R. Evans, A. Pritzel, T. Green, M. Figurnov, O. Ronneberger, K. Tunyasuvunakool, R. Bates, A. Zidek, A. Potapenko, A. Bridgland, C. Meyer, S. A. A. Kohl, A. J. Ballard, A. Cowie, B. Romera-Paredes, S. Nikolov, R. Jain, J. Adler, T. Back, S. Petersen, D. Reiman, E. Clancy, M. Zielinski, M. Steinegger, M. Pacholska, T. Berghammer, S. Bodenstein, D. Silver, O. Vinyals, A. W. Senior, K. Kavukcuoglu, P. Kohli and D. Hassabis (2021). "Highly accurate protein structure prediction with AlphaFold." Nature.

Kovacs, J. A., V. E. Galkin and W. Wriggers (2018). "Accurate flexible refinement of atomic models against medium-resolution cryo-EM maps using damped dynamics." BMC Struct Biol 18(1): 12. Kremer, J. R., D. N. Mastronarde and J. R. McIntosh (1996). "Computer visualization of threedimensional image data using IMOD." J Struct Biol 116(1): 71-76.

Kreutzberger, M. A. B., C. Ewing, F. Poly, F. Wang and E. H. Egelman (2020). "Atomic structure of the Campylobacter jejuni flagellar filament reveals how epsilon Proteobacteria escaped Toll-like receptor 5 surveillance." Proc Natl Acad Sci U S A 117(29): 16985-16991.

Kurniyati, K., J. F. Kelly, E. Vinogradov, A. Robotham, Y. Tu, J. Wang, J. Liu, S. M. Logan and C. Li (2017). "A novel glycan modifies the flagellar filament proteins of the oral bacterium Treponema denticola." Microbiol 103(1): 67-85.

Lambert, A., M. Picardeau, D. A. Haake, R. W. Sermswan, A. Srikram, B. Adler and G. A. Murray (2012). "FlaA proteins in Leptospira interrogans are essential for motility and virulence but are not required for formation of the flagellum sheath." Infect Immun 80(6): 2019-2025.

Leifson, E. (1960). "Atlas of bacterial flagellation."

Li, C., L. Corum, D. Morgan, E. L. Rosey, T. B. Stanton and N. W. Charon (2000). "The spirochete FlaA periplasmic flagellar sheath protein impacts flagellar helicity." J Bacteriol 182(23): 6698-6706.

Li, C., A. Motaleb, M. Sal, S. F. Goldstein and N. W. Charon (2000). "Spirochete periplasmic flagella and motility." J Mol Microbiol Biotechnol 2(4): 345-354.

Lin, M., O. Surujballi, K. Nielsen, S. Nadin-Davis and G. Randall (1997). "Identification of a 35-kilodalton serovar-cross-reactive flagellar protein, $\mathrm{FlaB}$, from Leptospira interrogans by $\mathrm{N}$-terminal sequencing, gene cloning, and sequence analysis." Infect Immun 65(10): 4355-4359.

Ludtke, S. J., P. R. Baldwin and W. Chiu (1999). "EMAN: semiautomated software for high-resolution single-particle reconstructions." J Struct Biol 128(1): 82-97.

Maki-Yonekura, S., K. Yonekura and K. Namba (2010). "Conformational change of flagellin for polymorphic supercoiling of the flagellar filament." Nat Struct Mol Biol 17(4): 417-422.

Malmstrom, J., M. Beck, A. Schmidt, V. Lange, E. W. Deutsch and R. Aebersold (2009). "Proteome-wide cellular protein concentrations of the human pathogen Leptospira interrogans." Nature 460(7256): 762765.

Mastronarde, D. N. (2005). "Automated electron microscope tomography using robust prediction of specimen movements." J Struct Biol 152(1): 36-51.

Mentes, A., A. Huehn, X. Liu, A. Zwolak, R. Dominguez, H. Shuman, E. M. Ostap and C. V. Sindelar (2018). "High-resolution cryo-EM structures of actin-bound myosin states reveal the mechanism of myosin force sensing." Proc Natl Acad Sci U S A 115(6): 1292-1297. 
Miller, M. R., K. A. Miller, J. Bian, M. E. James, S. Zhang, M. J. Lynch, P. S. Callery, J. M. Hettick, A. Cockburn, J. Liu, C. Li, B. R. Crane and N. W. Charon (2016). "Spirochaete flagella hook proteins selfcatalyse a lysinoalanine covalent crosslink for motility." Nat Microbiol 1(10): 16134.

Mitchison, M., J. I. Rood, S. Faine and B. Adler (1991). "Molecular analysis of a Leptospira borgpetersenii gene encoding an endoflagellar subunit protein." J Gen Microbiol 137(7): 1529-1536.

Montemayor, E. J., N. T. Ploscariu, J. C. Sanchez, D. Parrell, R. S. Dillard, C. W. Shebelut, Z. Ke, R. C. Guerrero-Ferreira and E. R. Wright (2021). "Flagellar Structures from the Bacterium Caulobacter crescentus and Implications for Phage varphi CbK Predation of Multiflagellin Bacteria." J Bacteriol 203(5).

Namba, K. and F. Vonderviszt (1997). "Molecular architecture of bacterial flagellum." Q Rev Biophys 30(1): 1-65.

Nauman, R. K., S. C. Holt and C. D. Cox (1969). "Purification, ultrastructure, and composition of axial filaments from Leptospira." J Bacteriol 98(1): 264-280.

Norris, S. J., N. W. Charon, R. G. Cook, M. D. Fuentes and R. J. Limberger (1988). "Antigenic relatedness and $\mathrm{N}$-terminal sequence homology define two classes of periplasmic flagellar proteins of Treponema pallidum subsp. pallidum and Treponema phagedenis." J Bacteriol 170(9): 4072-4082.

Paster, B. J. (2010). Phylum XV. Spirochaetes Garrity and Holt 2001. Bergey's Manual ${ }^{\circledR}$ of Systematic Bacteriology: Volume Four The Bacteroidetes, Spirochaetes, Tenericutes (Mollicutes), Acidobacteria, Fibrobacteres, Fusobacteria, Dictyoglomi, Gemmatimonadetes, Lentisphaerae, Verrucomicrobia, Chlamydiae, and Planctomycetes. N. R. Krieg, J. T. Staley, D. R. Brown et al. New York, NY, Springer New York: 471-566.

Pead, P. J. (1979). "Electron microscopy of Campylobacter jejuni." J Med Microbiol 12(3): 383-385. Perez-Riverol, Y., A. Csordas, J. Bai, M. Bernal-Llinares, S. Hewapathirana, D. J. Kundu, A. Inuganti, J. Griss, G. Mayer, M. Eisenacher, E. Perez, J. Uszkoreit, J. Pfeuffer, T. Sachsenberg, S. Yilmaz, S. Tiwary, J. Cox, E. Audain, M. Walzer, A. F. Jarnuczak, T. Ternent, A. Brazma and J. A. Vizcaino (2019). "The PRIDE database and related tools and resources in 2019: improving support for quantification data." Nucleic Acids Res 47(D1): D442-D450.

Picardeau, M. (2017). "Virulence of the zoonotic agent of leptospirosis: still terra incognita?" Nat Rev Microbiol 15(5): 297-307.

Picardeau, M., D. M. Bulach, C. Bouchier, R. L. Zuerner, N. Zidane, P. J. Wilson, S. Creno, E. S. Kuczek, S. Bommezzadri, J. C. Davis, A. McGrath, M. J. Johnson, C. Boursaux-Eude, T. Seemann, Z. Rouy, R. L. Coppel, J. I. Rood, A. Lajus, J. K. Davies, C. Medigue and B. Adler (2008). "Genome sequence of the saprophyte Leptospira biflexa provides insights into the evolution of Leptospira and the pathogenesis of leptospirosis." PLoS One 3(2): e1607.

Punjani, A., J. L. Rubinstein, D. J. Fleet and M. A. Brubaker (2017). "cryoSPARC: algorithms for rapid unsupervised cryo-EM structure determination." Nat Methods 14(3): 290-296.

Rossello, J., A. Lima, M. Gil, J. Rodriguez Duarte, A. Correa, P. C. Carvalho, A. Kierbel and R. Duran (2017). "The EAL-domain protein FcsR regulates flagella, chemotaxis and type III secretion system in Pseudomonas aeruginosa by a phosphodiesterase independent mechanism." Sci Rep 7(1): 10281. Rubinstein, J. L. and M. A. Brubaker (2015). "Alignment of cryo-EM movies of individual particles by optimization of image translations." J Struct Biol 192(2): 188-195.

Samanta, U. and P. Chakrabarti (2001). "Assessing the role of tryptophan residues in the binding site." Protein Eng 14(1): 7-15.

San Martin, F., A. E. Mechaly, N. Larrieux, E. A. Wunder, Jr., A. I. Ko, M. Picardeau, F. Trajtenberg and A. Buschiazzo (2017). "Crystallization of FcpA from Leptospira, a novel flagellar protein that is essential for pathogenesis." Acta Crystallogr F Struct Biol Commun 73(Pt 3): 123-129. 
Sasaki, Y., A. Kawamoto, H. Tahara, K. Kasuga, R. Sato, M. Ohnishi, S. Nakamura and N. Koizumi (2018). "Leptospiral flagellar sheath protein FcpA interacts with FlaA2 and FlaB1 in Leptospira biflexa." PLoS One 13(4): e0194923.

Tang, G., L. Peng, P. R. Baldwin, D. S. Mann, W. Jiang, I. Rees and S. J. Ludtke (2007). "EMAN2: an extensible image processing suite for electron microscopy." J Struct Biol 157(1): 38-46.

Trueba, G. A., C. A. Bolin and R. L. Zuerner (1992). "Characterization of the periplasmic flagellum proteins of Leptospira interrogans." J Bacteriol 174(14): 4761-4768.

Wagner, T., L. Lusnig, S. Pospich, M. Stabrin, F. Schonfeld and S. Raunser (2020). "Two particle-picking procedures for filamentous proteins: SPHIRE-crYOLO filament mode and SPHIRE-STRIPER." Acta Crystallogr D Struct Biol 76(Pt 7): 613-620.

Wagner, T. and S. Raunser (2020). "The evolution of SPHIRE-crYOLO particle picking and its application in automated cryo-EM processing workflows." Commun Biol 3(1): 61.

Wang, F., A. M. Burrage, S. Postel, R. E. Clark, A. Orlova, E. J. Sundberg, D. B. Kearns and E. H. Egelman (2017). "A structural model of flagellar filament switching across multiple bacterial species." Nat Commun 8(1): 960.

Wang, W., Z. Jiang, M. Westermann and L. Ping (2012). "Three mutations in Escherichia coli that generate transformable functional flagella." J Bacteriol 194(21): 5856-5863.

Wolgemuth, C. W. (2015). "Flagellar motility of the pathogenic spirochetes." Semin Cell Dev Biol 46: 104112.

Wolgemuth, C. W., N. W. Charon, S. F. Goldstein and R. E. Goldstein (2006). "The flagellar cytoskeleton of the spirochetes." J Mol Microbiol Biotechnol 11(3-5): 221-227.

Wriggers, W. (2012). "Conventions and workflows for using Situs." Acta Crystallogr D Biol Crystallogr 68(Pt 4): 344-351.

Wunder, E. A., H. Adhikarla, C. Hamond, K. A. Owers Bonner, L. Liang, C. B. Rodrigues, V. Bisht, J. E. Nally, D. P. Alt, M. G. Reis, P. J. Diggle, P. L. Felgner and A. Ko (2021). "A live attenuated-vaccine model confers cross-protective immunity against different species of the Leptospira genus." Elife 10.

Wunder, E. A., Jr., C. P. Figueira, N. Benaroudj, B. Hu, B. A. Tong, F. Trajtenberg, J. Liu, M. G. Reis, N. W. Charon, A. Buschiazzo, M. Picardeau and A. I. Ko (2016). "A novel flagellar sheath protein, FcpA, determines filament coiling, translational motility and virulence for the Leptospira spirochete." Mol Microbiol 101(3): 457-470.

Wunder, E. A., Jr., L. Slamti, D. N. Suwondo, K. H. Gibson, Z. Shang, C. V. Sindelar, F. Trajtenberg, A. Buschiazzo, A. I. Ko and M. Picardeau (2018). "FcpB Is a Surface Filament Protein of the Endoflagellum Required for the Motility of the Spirochete Leptospira." Front Cell Infect Microbiol 8: 130.

Wyss, C. (1998). "Flagellins, but not endoflagellar sheath proteins, of Treponema pallidum and of pathogen-related oral spirochetes are glycosylated." Infect Immun 66(12): 5751-5754.

Yonekura, K., S. Maki-Yonekura and K. Namba (2003). "Complete atomic model of the bacterial flagellar filament by electron cryomicroscopy." Nature 424(6949): 643-650.

Zhang, K. (2016). "Gctf: Real-time CTF determination and correction." J Struct Biol 193(1): 1-12.

Zheng, S. Q., E. Palovcak, J. P. Armache, K. A. Verba, Y. Cheng and D. A. Agard (2017). "MotionCor2: anisotropic correction of beam-induced motion for improved cryo-electron microscopy." Nat Methods 14(4): 331-332.

Zivanov, J., T. Nakane, B. O. Forsberg, D. Kimanius, W. J. Hagen, E. Lindahl and S. H. Scheres (2018). "New tools for automated high-resolution cryo-EM structure determination in RELION-3." Elife 7. Zivanov, J., T. Nakane and S. H. W. Scheres (2020). "Estimation of high-order aberrations and anisotropic magnification from cryo-EM data sets in RELION-3.1." IUCrJ 7(Pt 2): 253-267. 